\title{
Comparison Among Some Models of Orientation Selectivity
}

\author{
Andrew F. Teich and Ning Qian \\ Center for Neurobiology and Behavior, Mahoney Center for Brain and Behavior Research, and Department of Physiology \\ and Cellular Biophysics, Columbia University, New York, New York
}

Submitted 7 January 2005; accepted in final form 14 April 2006

Teich, Andrew F. and Ning Qian. Comparison among some models of orientation selectivity. J Neurophysiol 96: 404-419, 2006. First published April 19, 2006; doi:10.1152/jn.00015.2005. Several models exist for explaining primary visual cortex (V1) orientation tuning. The modified feedforward model (MFM) and the recurrent model (RM) are major examples. We have implemented these two models, at the same level of detail, alongside a few newer variations, and thoroughly compared their receptive-field structures. We found that antiphase inhibition in the MFM enhances both spatial phase information and orientation tuning, producing well-tuned simple cells. This remains true for a newer version of the MFM that incorporates untuned complex-cell inhibition. In contrast, when the recurrent connections in the RM are strong enough to produce typical V1 orientation tuning, they also eliminate spatial phase information, making the cells complex. Introducing phase specificity into the connections of the RM (as done in an original version of the RM) can make the cells phase sensitive, but the cells show an incorrect $90^{\circ}$ peak shift of orientation tuning under opposite contrast signs. An inhibition-dominant version of the RM can generate well-tuned cells across the simple-complex spectrum, but it predicts that the net effect of cortical interactions is to suppress feedforward excitation across all orientations in simple cells. Finally, adding antiphase inhibition used in the MFM into the RM produces a most general model. We call this new model the modified recurrent model (MRM) and show that this model can also produce well-tuned cells throughout the simple-complex spectrum. Unlike the inhibition-dominant RM, the MRM is consistent with data from cat $\mathrm{V} 1$, suggesting that the net effect of cortical interactions is to boost simple cell responses at the preferred orientation. These results suggest that the MFM is well suited for explaining orientation tuning in simple cells, whereas the standard RM is for complex cells. The assignment of the RM to complex cells also avoids conflicts between the RM and the experiments of cortical inactivation (done on simple cells) and the spatial-frequency dependency of orientation tuning (found in simple cells). Because orientation-tuned V1 cells show a continuum of simple- to complex-cell behavior, the MRM provides the best description of V1 data.

\section{N T R O D U C T I O N}

Strong orientation tuning first emerges in primary visual cortex (V1) (Hubel and Wiesel 1962). However, despite decades of studies and progress, the exact mechanism underlying the phenomenon remains controversial. Hubel and Wiesel initially proposed that orientation tuning of a V1 cell is established by receiving feedforward inputs from several lateral geniculate nucleus (LGN) cells with properly aligned, centersurround receptive fields. The oriented ON and OFF subregions of a V1 cell are assumed to arise from the on-center and OFF-center LGN cells, respectively. With a sufficiently large aspect ratio (the length-to-width ratio of a subregion) and a

Address for reprint requests and other correspondence: A. Teich, Center for Neurobiology and Behavior, Columbia University, Annex Room 521, 1051 Riverside Drive, New York, NY 10032 (E-mail: aft25@columbia.edu). high spiking threshold to remove responses from nonpreferred orientations, the V1 cell can have sharp orientation tuning as observed. Although the proposed LGN-to-V1 connectivity pattern has been supported by experimental evidence (Chapman et al. 1991; Reid and Alonso 1995; Tanaka 1983), this feedforward model cannot be the whole story because it has difficulties with other observations, especially the contrast invariance of orientation tuning (Sclar and Freeman 1982; Skottun et al. 1987) and the effects of cortical inhibition blockade (Sillito 1975; Sillito et al. 1980; Tsumoto et al. 1979; for thorough reviews also see Ferster and Miller 2000; Somers et al. 1995, Sompolinsky and Shapley 1997). Contrast invariance refers to the fact that the orientation tuning width of a cell does not change much with the stimulus contrast. The feedforward model predicts broadening of tuning with increasing contrast because a cell's membrane potential evoked by nonoptimal orientations should increase with contrast and is therefore more likely to reach the firing threshold at higher contrasts. Anderson et al. (2000a) recently found that neuronal noise explains why a contrast-invariant membrane potential leads to a contrast-invariant firing rate. However, this important result does not solve the problem of the feedforward model because the model cannot explain contrast invariance of the membrane potential in the first place. As the authors indicate, a cortical contribution is required to completely explain contrast invariance.

To resolve these problems, several groups proposed the recurrent model (RM), which assumes a weak feedforward orientation bias (i.e., a small aspect ratio) by the mechanism of Hubel and Wiesel and a subsequent sharpening of the tuning by both recurrent excitation and inhibition among the V1 cells (Ben-Yishai et al. 1995; Carandini and Ringach 1997; Douglas et al. 1995; Somers et al. 1995). The RM can account for an impressively large amount of experimental data (Somers et al. 1995; Sompolinsky and Shapley 1997), including the contrast invariance and the effects of inhibition blockade. However, it has difficulties with more recent cortical inactivation experiments (Chung and Ferster 1998; Ferster et al. 1996). By silencing cortical spiking activities through cooling or electrical shock and thereby greatly reducing intracortical interactions, Ferster and coworkers found that the intracellularly recorded orientation tuning curves of simple cells in the input layer of cat V1 did not become broader than those under the normal condition (Chung and Ferster 1998; Ferster et al. 1996). Another problem with the RM is that the width of tuning in the model is largely determined by the strong intracortical recurrent interactions. Consequently, the model cannot explain the

\footnotetext{
The costs of publication of this article were defrayed in part by the payment of page charges. The article must therefore be hereby marked "advertisement" in accordance with 18 U.S.C. Section 1734 solely to indicate this fact.
} 
fact that the orientation tuning width of simple cells decreases with increasing spatial frequency of a grating stimulus (Vidyasagar and Siguenza 1985).

These problems of the RM prompted Troyer et al. (1998) to propose a new modification of Hubel and Wiesel's feedforward model. According to Troyer et al., V1 orientation tuning mainly results from the feedforward mechanism and the contrast invariance is maintained by cortical inhibition, which is strongest between simple cells with opposite receptive-field polarities. This inhibition is termed "antiphase" inhibition, and there is partial evidence for it from intracellular recordings (Anderson et al. 2000b; Ferster 1988; Hirsch et al. 1998). The full version of the model also incorporates intracortical recurrent interactions for amplifying neuronal responses, but not for sharpening the bandwidth of feedforward tuning. In the rest of this paper, we will follow Ferster and Miller (2000) to refer to this model as the modified feedforward model (MFM). Although successful in many ways (Kayser et al. 2001; Krukowski and Miller 2001; Troyer et al. 1998), the MFM has more difficulties than the RM in explaining temporal dynamics (Pei et al. 1994; Ringach et al. 1997, 2003; Shapley et al. 2003; Shevelev et al. 1993, 1998) and the learning- and adaptationinduced plasticity of orientation tuning (Dragoi et al. 2002; Felsen et al. 2002; Teich and Qian 2003).

There is a new variant of the MFM (Lauritzen and Miller 2003) that includes untuned complex-cell inhibition, in addition to the antiphase inhibition, for the maintenance of contrast invariance. There is also a new inhibition-dominant variant of the RM (McLaughlin et al. 2000), such that V1 recurrent interactions suppress a cell's response at every stimulus orientation. We have examined these interesting variants together with the original RM and MFM in our study. However, it is more logical for our presentation to first focus on the original RM and MFM before moving on to their variants.

Both the RM and the MFM contain orientation-tuned feedforward input (as suggested by Hubel and Wiesel) and recurrent intracortical interactions. However, there are major differences between them (see METHODS), and these differences lead to divergent properties of the models, as we show in this paper. It is difficult to compare the models fairly based on the published studies because different studies often implement the models with very different degrees of complexity. We therefore implemented the RM and MFM at the same level of detail in our comparison. In particular, we investigated the possibility that the MFM and RM may be better suited for explaining strong orientation tuning in simple and complex cells, respectively. This is an interesting possibility because, if true, it helps alleviate the above-mentioned conflicts between the models and some experimental data (see DISCUSSION). Another difficulty with comparing the models is that a given result can sometimes be described differently. For example, antiphase inhibition in the MFM removes the contrast-dependent DC responses across all orientations (Troyer et al. 1998). This result can be described as either the presence or the absence of cortical sharpening of orientation tuning in the MFM depending on whether one includes or excludes the DC responses in the tuning-width calculation. Similarly, one can emphasize either the same-orientation or the orthogonal-orientation inhibition generated by broadly tuned antiphase inhibition. We will be as specific as possible when comparing models herein.
Both the RM and MFM were originally proposed as orientation mechanisms for simple cells (Somers et al. 1995; Troyer et al. 1998). The MFM is applicable only to simple cells because the connections in the model are based on receptivefield phases (Troyer et al. 1998). (Of course, one can assume that complex cells inherit their sharp orientation tuning from the simple cells described by the MFM, but such complex cells are unlikely to explain the observed orientation plasticity induced by learning or adaptation; Felsen et al. 2002; Teich and Qian 2003; Teich and Qian, unpublished observations.) In contrast, the RM is applicable to complex cells because the connections are independent of receptive-field phases. However, it is not clear whether the RM really works for simple cells as well. On the one hand, Chance et al. (1999) showed that strong recurrent interactions lead to complex-cell behavior in a related model on frequency tuning. On the other hand, the V1 cells in the RM of Somers et al. (1995) did seem to maintain discrete ON and OFF subregions despite the strong recurrent interactions, suggesting that the recurrent model should work for simple cells. Other implementations of the RM do not bear on this issue because they are more abstract and do not explicitly include receptive fields (Ben-Yishai et al. 1995; Carandini and Ringach 1997; Douglas et al. 1995; Teich and Qian 2003).

A close examination of Somers et al.'s model reveals that they made the simplifying assumption that all model cells have the same even receptive-field phase (but different orientations). Real V1 cells, however, cover a broad range of receptive-field phases. If the strong recurrent connections are introduced among V1 cells with different phases (determined by the feedforward inputs) to generate sharp orientation tuning, can the cells in such a multiphase RM maintain their phase sensitivity? A related question is: Do phase-sensitive cells in Somers et al.'s single-phase RM really behave like simple cells? Furthermore, because there is also recurrent excitation in the MFM, do cells in that model maintain their simple-cell identity? We address these questions in this study. We then examined the receptive-field structure of the two variant models mentioned above. Finally, we combined the RM and the MFM into a new model, the modified recurrent model (MRM), and investigated whether the new model provides the best description of orientation tuning in V1 where cells have various degrees of simple to complex behavior. Preliminary results were previously reported in abstract form (Teich and Qian 2004).

\section{METHODS}

We first implemented the RM and the MFM at an equal level of detail and compared the responses of the two models to drifting gratings and flashed stationary bars. These responses would allow us to determine whether a model cell behaves like a simple or complex cell. Our implementation of the MFM was based on Troyer et al. (1998), who simulated both a very simple "conceptual" model and a more detailed "computational" model. We implemented their computational model but replaced the spiking neurons with rate-coding neurons; this simplification greatly improves computational efficiency without altering the essential features of the original model. Their conceptual model is not sufficient for our purpose because it has only two receptive-field phases and does not include recurrent cortical excitation; we wanted to know whether the MFM cells could behave like simple cells in a multiphase implementation with recurrent 
excitation. After constructing the MFM, we then built the RM at the same level of complexity. Both models start with a field of LGN cells, and the LGN response to a stimulus is then fed to a cortical network of V1 cells. The LGN cells are modeled the same way in both models and will be described first. The main difference between the two models is found in the aspect ratio of the thalamocortical connections and at the connectivity patterns among the V1 cells (detailed below). After implementing the RM and the MFM, we then considered the MFM with complex inhibition, the inhibition-dominant RM, and the MRM. All simulations were performed with Matlab (The MathWorks, Natick, MA) on a Linux computer.

\section{Stimuli}

We used drifting sinusoidal gratings and stationary bars to probe whether the cells in a model behave like simple or complex cells. The drifting gratings had the form

$$
\mathrm{S}(x, y, t)=\cos \left(w_{x} x+w_{y} y+w_{t} t\right)
$$

where $w_{t}$ represents the temporal frequency and $w_{x}$ and $w_{y}$ represent the spatial frequencies in the $x$ and $y$ dimensions, respectively. We did not explicitly include constant background luminance and contrast in the equation because the former would be filtered out by the LGN receptive fields whose DC responses were near zero, and the latter would be determined by scaling according to contrast response of real LGN cells (see following text). In our simulations, $w_{t} /(2 \pi)=2 \mathrm{~Hz}$, $w_{x}=w \cos \theta$, and $w_{y}=w \sin \theta$, where $w /(2 \pi)=0.8$ cycles $/$ deg and $\theta$ is the grating orientation ( 0 means vertical). The bars had a width of 30 arc min and a length spanning the entire receptive fields, and were turned on for $400 \mathrm{~ms}$ and off for $300 \mathrm{~ms}$. Thus for a vertical bar stimulus, $S(x, y, t)=1$ for $-15 \leq x \leq 15$ and $0 \leq t \leq 400$ (where $x$ is in arc minutes and $t$ is in milliseconds), and $S(x, y, t)=-1$, otherwise. Other bar orientations were obtained by rotation.

\section{LGN responses}

The LGN cells in both models closely follow the design of Troyer et al. (1998) but have a more convenient temporal response function. We considered 240 oN-center and 240 ofF-center cells. The cells of each type are arranged in a rectangular grid to provide inputs to vertically oriented V1 cells; feedforward inputs to V1 cells of other orientations were computed by proper rotations of the inputs to the vertical cells. Before rotation, centers of LGN cells were spaced 9 min apart along the vertical axis and 6 min apart along the horizontal axis. There was no spatial offset between the ON and OFF grids. Each cell's spatial receptive field is center-surround, modeled with a difference of Gaussians

$$
f(r)=\left(17 / \sigma_{\text {center }}^{2}\right) \mathrm{e}^{-r^{2} / \sigma_{\text {center }}^{2}}-\left(16 / \sigma_{\text {surround }}^{2}\right) e^{-r^{2} / \sigma_{\text {surround }}^{2}}
$$

where $r$ is the distance in any direction from the center of the receptive field, $\sigma_{\text {center }}=15^{\prime}$ and $\sigma_{\text {surround }}=60^{\prime}$. The temporal response function of the LGN cells takes the form (Chen et al. 2001)

$$
h(t)=\frac{t}{\tau^{2}} \exp \left(-\frac{t}{\tau^{2}}\right) \cos \left(\omega_{t}^{o} t+\phi_{t}\right)
$$

for $t \geq 0$ and $h(t)=0$, otherwise. Here $\tau$ determines the duration of the temporal envelope, and the sinusoidal term with frequency $\omega_{t}^{o}$ generates excitatory and inhibitory responses. Because for many real cells the first half-cycle of the temporal response is shorter by various amounts than the second half-cycle, the parameter $\phi_{t}$ is introduced to reduce the length of the first half-cycle (because of the rapid decay of the exponential, the durations of the third and later half-cycles are not important). In this paper, we let $\tau=16 \mathrm{~ms}, \omega_{t}^{o} /(2 \pi)=4 \mathrm{~Hz}$, and $\phi_{t}$ $=0.24$. The overall duration of this temporal response function is about $140 \mathrm{~ms}$. The resulting temporal response curve was consistent with physiologically reported temporal responses in LGN cells
(Gielen et al. 1981; Saul and Humphrey 1990). We also added a 50-ms delay to V1 cells' responses without explicitly simulating random individual synaptic delays.

The LGN response $R_{\text {lgn }}$ to a given stimulus was first determined through spatiotemporal convolutions of the stimulus $S(x, y, t)$ with the kernel $f(r) h(t)$

$$
R_{l g n}=S(x, y, t) * f(r) * h(t)
$$

and then scaled according to a contrast response curve. LGN responses to a drifting grating of contrast $C$ were scaled so that the amplitude of the F1 component follows the contrast response curve from Troyer et al. (1998): $R=R_{\max } \mathrm{C}^{n} /\left(C_{50}^{n}+C^{n}\right)$, where $R_{\max }=53$ spikes/s, $n=1.2$, and $C_{50}=13.3 \%$ for on-center cells; $R_{\max }=48.6$ spikes/s, $n=1.29$, and $C_{50}=7.18 \%$ for OFF-center cells. These contrast response functions assume stimulation at the optimal spatial frequency for the LGN filter (about 0.55 cycles/deg). LGN responses to nonoptimal frequencies were scaled down proportionally using the ratio of the filtered responses to the nonoptimal and the optimal frequencies. We assumed that the LGN contrast response curve to a stationary bar would have the same shape as the F1 curves for gratings. Because LGN responses to a flashed spot do not vary significantly between ON- and OFF-center cells (Saul and Humphrey 1990), we used an average $n$ value of 1.245 and an average $C_{50}$ value of 10.24 for both types of cells. We scaled the curve's amplitude by letting $R_{\max }=285$ spikes/s so that the initial peak response to a flashed bar of $50 \%$ contrast and optimal width was about 250 spikes/s (Tanaka 1983). Because the background firing rate is not included in the contrast responses functions, we added a background firing rate of 10 spikes/s for ON-center cells and 15 spikes/s for OFF-center cells after scaling [values taken from Troyer et al. (1998)].

\section{Cortical receptive fields}

The LGN inputs to a V1 cell were determined by a two-dimensional Gabor connectivity function (a Gaussian multiplied by a sinusoid). For vertically oriented V1 cells, it had the form

$$
g(x, y)=\exp \left\{-\left[x^{2} /\left(2 \sigma_{x}^{2}\right)\right]-\left[y^{2} /\left(2 \sigma_{y}^{2}\right)\right] \times \cos \left(w_{g} x+\phi\right)\right.
$$

where $w_{g} /(2 \pi)=0.8$ cycles/deg, $\phi$ determined the V1 receptive-field phase, and $\sigma_{x}$ and $\sigma_{y}$ determined the number of subregions and the aspect ratio (see following text). For V1 cells with orientation $\theta$, the Gabor connectivity function $g(x, y, \theta)$ was obtained from $g(x, y)$ by proper rotation. All LGN-to-V1 connections are excitatory. The positive values of the Gabor function represented excitatory connections from oN-center LGN cells and negative values represented excitatory connections from OFF-center LGN cells; in either case, the strength of the connection was represented by the absolute value of the Gabor at a given point. The Gaussian was elongated in the preferred orientation of the V1 cell under consideration and the sinusoid had the same orientation. The width and length of the Gaussian were defined by the $5 \%$ points of the peak, along the short and long axes of the Gaussian, respectively. The number of subregions was determined by the ratio of the Gaussian width to the width of a half-cycle of the sinusoid. The aspect ratio was defined as the ratio of the Gaussian length to the width of a half-cycle of the sinusoid. As noted in Eq. 5, we used a sinusoid with a half-cycle width of $0.625^{\circ}$, corresponding to a spatial frequency of 0.8 cycles/deg [roughly the mean preferred spatial frequency of cat cortical cells at $5^{\circ}$ eccentricity (Movshon et al. 1978b); all spatial dimensions can be scaled down to represent monkey V1 cells without affecting our conclusions]. The MFM and RM used the high and low values of the reported aspect ratios, respectively. For the MFM, we picked the Gaussian envelope to match the physiological receptive fields reported by Jones and Palmer (1987) and used by Troyer et al. (1998): 2.65 subregions and an aspect ratio of 4.54 (Fig. 1A1). These values yielded a feedforward input well tuned for orientation (Fig. 1A2). For the RM, we also had 2.65 
A1
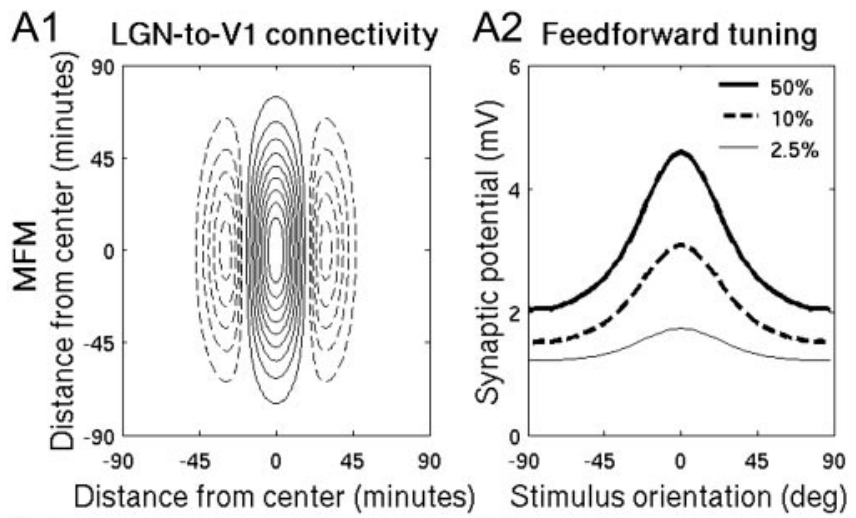

B1
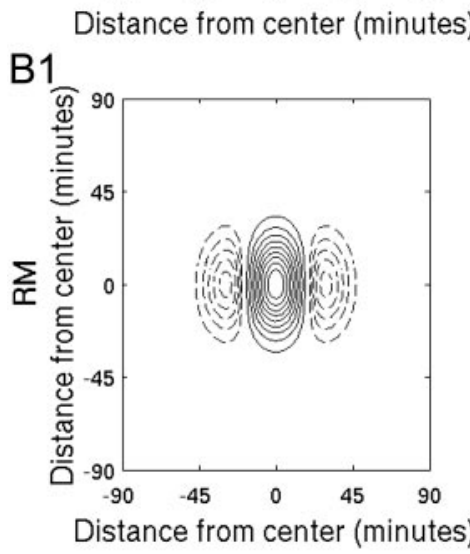

B2

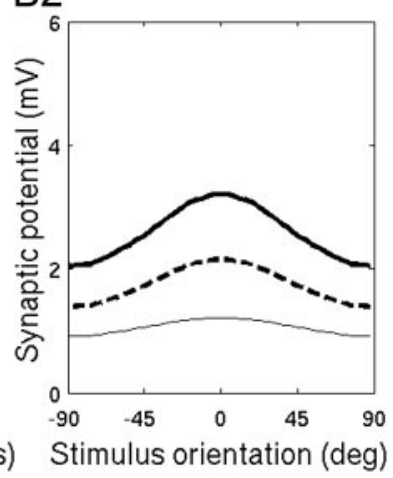

subregions but lowered the aspect ratio to 2 (Fig. 1B1), which is in line with the value used by Somers et al. (1995) and is close to the value of 1.7 reported by Pei et al. (1994). This yielded a broadly tuned feedforward input in Fig. $1 B 2$.

\section{Features common to the MFM and the RM}

Both the MFM and the RM have cortical excitatory cells and cortical inhibitory cells at eight evenly spaced spatial phases over $2 \pi$ and 64 evenly distributed orientations over $180^{\circ}$, for a total of 512 excitatory cells and 512 inhibitory cells. In the real cortex, excitatory cells outnumber inhibitory cells by a factor of three. For computational efficiency, we did not explicitly model this fact; instead, we simply let each excitatory cell in our models represent three cells of the same type. The connections from an excitatory cell to any cell represent the combined strengths of the three cells. The model cells are assumed to represent the same area of visual space and have retinotopically overlapping receptive fields (they draw feedforward input from the same field of LGN cells). Each cortical cell receives feedforward input from its LGN afferents as well as cortical input from other V1 cells. The nature of the intracortical connections depended on which model we used and is detailed below. For a given V1 cell, the connections it received were divided into three sets: the connections from LGN cells, the connections from excitatory V1 cells, and the connections from inhibitory V1 cells. We first normalized each set of the connections such that the sum of all connections within the set was one. We then multiplied all connections within a set by a common weighting factor, and different weighting factors were applied to different sets to manipulate their relative strengths. Because the recipient V1 cell can be either excitatory or inhibitory, there are a total of six weighting factors denoted by $e \rightarrow e, e \rightarrow i, i \rightarrow e, i \rightarrow i$, $F \rightarrow e$, and $F \rightarrow i$, which represent weights for excitatory to excitatory, excitatory to inhibitory, inhibitory to excitatory, inhibitory to inhibitory, feedforward to excitatory, and feedforward to inhibitory connections, respectively.

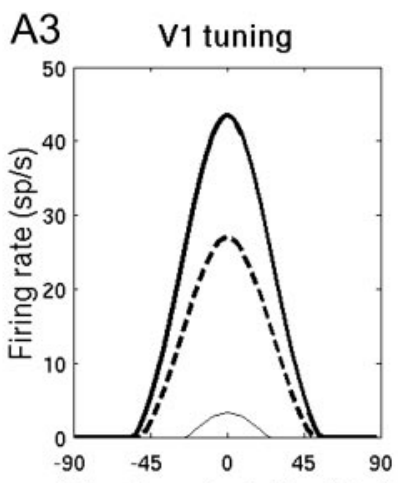

Stimulus orientation (deg)

B3

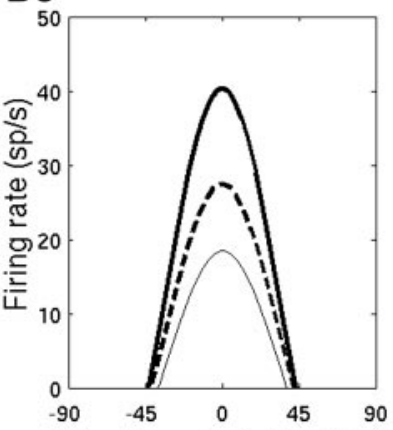

Stimulus orientation (deg)
FIG. 1. Contrast-invariant orientation tuning of our implementations of the modified feedforward model (MFM, top row) and the recurrent model (RM, bottom row). In the MFM, the lateral geniculate nucleus (LGN) to primary visual cortex (V1) connectivity pattern is assumed to have a long orientation axis (aspect ratio $=4.54 ; A 1$ ), which yields a feedforward input well tuned for orientation (A2). In $A 1$ and $B 1$, solid lines represent connections from ON-center LGN cells, whereas dotted lines represent connections from OFF-center LGN cells. DC baseline component in the feedforward input (A2) is eliminated through cortical antiphase inhibition, yielding contrast invariance $(A 3)$. Although the V1 output at the lowest contrast $(2.5 \%)$ is actually narrower than higher contrasts in the MFM, this problem vanishes when noise is introduced to the system (Troyer et al. 2002), and does not affect our results (which are at 50\% contrasts) in the rest of the paper. RM has a shorter axis of orientation (aspect ratio $=2 ; B 1$ ), which yields a feedforward input broadly tuned for orientation (B2). Intracortical connections both eliminate the DC baseline component in the feedforward input and sharpen the tuned component, yielding sharp, contrastinvariant tuning (B3).

The membrane potential $(V)$ of each cortical cell was updated according to the differential equation (Carandini and Ringach 1997; Teich and Qian 2003)

$$
\tau \partial V / \partial t+V=V_{f}+V_{e}-\mathrm{V}_{\mathrm{i}}
$$

where $\tau$ is the membrane time constant ( $15 \mathrm{~ms}$ in all simulations), and $V_{\mathrm{f}}, V_{\mathrm{e}}$, and $V_{\mathrm{i}}$ are the synaptic potentials generated by the feedforward, cortical excitatory, and cortical inhibitory inputs to the cell, respectively. For example, for an excitatory cell with orientation $\theta$, these three values were given by

$$
\begin{gathered}
V_{f}=(F \rightarrow e) \times \int_{-\infty}^{\infty} \int_{-\infty}^{\infty} g(x, y, \theta) \times R_{l g n}(x, y) \mathrm{d} x \mathrm{~d} y \\
V_{e}=(e \rightarrow e) \times \int_{-\pi / 2}^{\pi / 2} C_{e e}\left(\theta-\theta^{\prime}\right) R_{e}\left(\theta^{\prime}\right) \mathrm{d} \theta^{\prime} \\
V_{1}=(i \rightarrow e) \times \int_{-\pi / 2}^{\pi / 2} C_{i e}\left(\theta-\theta^{\prime}\right) R_{i}\left(\theta^{\prime}\right) \mathrm{d} \theta^{\prime}
\end{gathered}
$$

where $R_{\mathrm{lgn}}, R_{\mathrm{e}}$, and $R_{\mathrm{i}}$ are the firing rates of LGN cells, excitatory V1 cells, and inhibitory V1 cells, respectively; $C_{\text {ee }}$ and $C_{\text {ie }}$ represent the normalized connectivity strengths from excitatory and inhibitory V1 cells to the excitatory $\mathrm{V} 1$ cell under consideration, and are determined differently for each model (see details below). Similar equations can be written for each inhibitory $\mathrm{V} 1$ cell. The interval $(\Delta t)$ at which we integrated Eq. 6 was $1 \mathrm{~ms}$ in all simulations. Our models directly relate membrane potential to firing rate by the threshold function with a gain factor $\alpha$ (Carandini and Ringach 1997; Teich and Qian 2003)

$$
R=\alpha \max (V, 0)
$$

The $\alpha$ value was 5 for excitatory cells, 8 for inhibitory cells in our MFM [to give inhibitory cells a higher firing rate as in Troyer et al. (1998)], and 6.5 for all cortical cells in our RM. 


\section{Intracortical circuitry for the MFM}

Following Troyer et al. (1998), we determined the connection between any two cortical cells $a$ and $b$ by computing the normalized correlation between their receptive fields

$$
c(a, b)=c^{\prime}(a, b) / \sqrt{c^{\prime}(a, a) c^{\prime}(b, b)}
$$

where $c^{\prime}(a, b)$ is the raw correlation computed by multiplying the two Gabor connectivity functions (feedforward receptive fields) $g(x, y, \theta)$ of cells $a$ and $b$ point by point and then summing across all points. Two cortical cells with the exact same orientation and spatial phase have a normalized correlation of 1 and thus have the strongest positive (excitatory) connection; two cortical cells with the exact same orientation and opposite spatial phase (antiphase) have a normalized correlation of -1 and have the strongest negative (inhibitory) connection. All other combinations are intermediate, with the nature of the connection (excitatory or inhibitory) being determined by the sign of the normalized correlation, and the strength of the connection being determined by the absolute value of the normalized correlation raised to a power Npow. Mathematically, the connection strength $C(a \rightarrow b)$ from cell $a$ to cell $b$ is based on the normalized correlation according to

$$
C(a \rightarrow b)=[\operatorname{sgn}(a) c(a, b)]_{+}{ }^{N p o w}
$$

where $\operatorname{sgn}(a)=1$ if $a$ is excitatory and -1 if $a$ is inhibitory, and $[x]_{+}=x$ if $x>0$ and 0 , otherwise. Npow determines the strength of connectivity as a function of correlation; larger values lead to less connectivity between cells that are weakly correlated. We used an Npow value of 6, which is the default value used in Troyer et al. (1998).

As we mentioned earlier, after normalizing the feedforward, cortical excitatory, and cortical inhibitory connections to a given cell separately, each connection type was then weighted. In the MFM, $e \rightarrow$ $e$ was set to $0.13, e \rightarrow i$ was set to $0.15, i \rightarrow e$ was set to $0.22, i \rightarrow$ $i$ was set to 0 , and $F \rightarrow e$ and $F \rightarrow i$ were set to 0.1 . The background $\mathrm{V} 1$ activity resulting from the LGN input in the absence of a stimulus was 0 spikes/s for excitatory cells and 10 spikes/s for inhibitory cells. Note that for the MFM, $i \rightarrow i$ was set to 0 as in Troyer et al. (1998); a nonzero connection among the inhibitory cells will reduce their activities across all orientations and thus make antiphase inhibition to excitatory cells less effective.

Anatomical studies have suggested that thalamic inputs constitute a small percentage of the total inputs to V1 simple cells, and that among cortical inputs, excitatory synapses are more prevalent than inhibitory synapses (Ahmed et al. 1994; Gabbott and Somogyi 1986; Peters and Payne 1993). On the other hand, physiological studies showed that V1 responses to thalamic input are more powerful than to cortical input (Stratford et al. 1996). In addition, cortical inhibitory synapses are closer to the soma (Douglas and Martin 2004) and are thus probably more powerful than cortical excitatory synapses. We did not explicitly simulate these facts for the sake of computational efficiency. Instead, we can simply assume that when comparing the feedforward connection strengths with intracortical connection strengths, each intracortical connection should be viewed as the combined effect of many synapses. Likewise, when comparing the intracortical excitatory connections with inhibitory connections, each excitatory connection should be viewed as the combined connections from a few excitatory cells. The same comments also apply to all of the other models discussed in the following text.

\section{The MFM with complex-cell inhibition}

In addition to the standard MFM, we also ran a newer version of the MFM with complex-cell inhibition (Lauritzen and Miller 2003). This involved applying the same untuned, phase-insensitive inhibition to every cortical cell. All of the parameters for the MFM with complex inhibition are identical to the standard MFM except for the connection strengths between different cell types and Npow. The connection strengths were as follows: $e \rightarrow e$ was set to $0.16, e \rightarrow i$ was set to 0.18 , $i \rightarrow e$ was set to $0.25, i \rightarrow i$ was set to 0.1 , and $F \rightarrow e$ and $F \rightarrow i$ were set to 0.1. Npow was set to 24. These adjustments were made to accommodate added complex inhibition. In addition, $c i \rightarrow e$ was set to $1.2, c i \rightarrow i$ was set to 1.2 , and $F \rightarrow c i$ was set to 0.1 , where $c i$ represents untuned, complex inhibition. Because complex inhibition is applied equally to every cell, we had only one inhibitory complex cell in every simulation and simply applied its output to every other cortical cell at each time step.

\section{Intracortical circuitry for the RM}

Unlike the MFM, the connections between two cells in the standard RM were independent of the cells' receptive field phase relationship and were determined only by the difference in the cells' preferred orientations. We used Gaussians to describe the connection strengths as a function of the difference in the cells' preferred orientations. The connections from an excitatory cell to any cell (the excitatory profile) are given by a Gaussian with SD of $35^{\circ}$. The connections from an inhibitory cell to any cell (the inhibitory profile) are given by a Gaussian with SD of $52^{\circ}$. When these two profiles are subtracted from each other, the resulting net recurrent interaction as a function of preferred orientation difference has the "Mexican-hat" shape, a prominent feature of the RM. Also unlike the MFM, there are direct connections among the inhibitory cells.

Our previous work with the RM (Teich and Qian 2003) followed the study of Ferster (1986) in assuming that stimuli orthogonal to a cell's preferred orientation do not evoke either excitatory or inhibitory postsynaptic potentials. However, in this study we discovered that once multiple phases are included, the RM requires at least some net inhibition at orthogonal orientations to avoid spurious peaks at orthogonal orientations. The reason can be understood by considering two cells with the same receptive-field phase that are oriented $90^{\circ}$ away from one another. When a light bar is aligned with the oN subregion of one cell and thus generating maximum feedforward input to the cell, the second, orthogonal cell receives minimum feedforward input for this stimulus (see solid curve in Fig. 4A). When a dark bar is aligned with the on subregion of the first cell, the first cell receives minimum feedforward input, whereas the orthogonal cell receives maximum feedforward input (see dashed curve in Fig. 4A). This orthogonal peak in the feedforward input to the second cell will lead to a spurious peak in its orientation tuning unless there is some stimulus-driven inhibition at the orthogonal orientation. There is also experimental evidence supporting the presence of orthogonal inhibition (Allison et al. 2001; Bonds 1989; Martinez et al. 2002; Monier et al. 2003; Shapley et al. 2003). In particular, Monier et al. (2003) found that in one fifth of their intracellular recordings, inhibition peaked at the orthogonal orientation, and some evidence for this was also found by Martinez et al. (2002) in layer 5. Note that the MFM also requires inhibition at orthogonal orientations (see DISCUSSION).

For the RM, $e \rightarrow e$ and $e \rightarrow i$ were $1.6, i \rightarrow e$ and $i \rightarrow i$ were 1.8 , and $F \rightarrow e$ and $F \rightarrow i$ were 0.07 . With these parameters, the background V1 activity in the absence of a stimulus was 2.47 spikes/s for all cortical cells. Figure 1 confirms that our multiphase implementations of the MFM and the RM work well in generating contrastinvariant orientation tuning curves (Fig. 1, A3 and B3) as observed in V1.

In the preceding implementation of the $\mathrm{RM}$, the $\mathrm{V} 1$ cells' receptive fields had eight different spatial phases (determined by the feedforward LGN inputs) just as in the MFM. To compare with the original single-phase RM of Somers et al. (1995), we also considered a single-phase version of our RM by letting the eight cells at each orientation all have the same even spatial phase. In this single-phase $R M$, the values we used for $e \rightarrow e$ and $e \rightarrow i$ were 1.55 , and $i \rightarrow e$, $i \rightarrow i, F \rightarrow e$, and $F \rightarrow i$ were identical to the values in the multiphase 
RM. The background V1 activity in the absence of a stimulus was 2 spikes/s for all cortical cells in the single-phase RM.

\section{Differences between the MFM and the RM}

The terms recurrent model and modified feedforward model may be a little misleading because both models contain orientation-tuned feedforward input and recurrent intracortical interactions. However, there are major differences between them, as we alluded to above. To make the discussions more precise, note that for both models, the feedforward input to a V1 cell can be viewed as a sum of a DC component (the constant baseline response across all orientations) and a tuned component (the orientation-dependent portion) (Fig. 1, A2 and $B 2)$. The DC component equals the response at the orientation orthogonal to the cell's preferred orientation. By subtracting this component from the total feedforward input, one obtains the tuned component. Both components grow with the stimulus contrast (Fig. 1, $A 2$ and $B 2$ ) because a V1 cell receives inputs from both oN-center and OFF-center LGN cells, and as the stimulus intensity increases relative to the background, the OFF-center cell firing rates quickly drop to zero and cannot go any lower to counter the growing on-center cell responses before they saturate (Shapley et al. 2003; Troyer et al. 1998).

There are several crucial differences between the two models. Most prominently, the RM assumes that the tuned component of the feedforward input is broad and is sharpened by intracortical interactions, whereas the MFM assumes that the tuned component in the feedforward input is narrow and is not sharpened further by intracortical connections. These different feedforward tuning curves arise from different assumptions about the aspect ratio of the LGN-to-V1 projections; the RM assumes a small aspect ratio, whereas the MFM assumes a large one.

The intracortical processing is necessary for both models because, without it, the V1 tuning would be similar to the feedforward tuning, with a DC component (across all orientations) and a tuned component. Because these components grow with contrast, a fixed firing threshold cannot make the V1 tuning contrast invariant. In the RM, the intracortical interactions both sharpen the tuned component and remove the DC component of the feedforward input (Fig. 1B3). However, according to the MFM, the intracortical interactions do not sharpen the tuned component further but only remove the DC component (Fig. 1A3).

These different requirements are realized by different intracortical connection patterns used in the two models: 1) The connections in the MFM depend on both the orientation difference and the receptivefield phase difference between two V1 cells. In the RM, the connections are only orientation dependent but not phase dependent. 2) Both models assume that the connection strengths fall off with increasing difference between two cells' preferred orientations. However, the $\mathrm{RM}$ assumes that the inhibitory connections cover a wider range of orientation differences than that of the excitatory connections, resulting in a Mexican-hat net interaction profile, whereas the MFM assumes equal width of the excitatory and inhibitory profiles. In the $\mathrm{RM}$, the inhibitory connections among cells tuned to very different orientations suppress responses to the nonpreferred orientations including the orthogonal orientation. In the MFM, the antiphase inhibition among cells with similar receptive-field orientation (but opposite receptive-field phases) does the same job. Although the inhibitory connections in the MFM do not cover a broader range of orientation differences than the excitatory connections, the inhibitory cells' responses are assumed to have a broad, contrast-dependent DC component, which suppresses the excitatory cells' responses at the nonpreferred orientations including the orthogonal orientation (Troyer et al. 1998). So strictly speaking, orthogonal inhibition is also present in the MFM. 3) There are direct connections among the inhibitory cells in the RM; such connections are not allowed in the MFM because they would diminish the antiphase inhibition mentioned above. Antiphase inhibition has also been called feedforward inhibition (Ferster and Miller 2000), although the inhibitory cells do receive recurrent cortical inputs.

There is also a quantitative difference between the two models: cortical weights are an order of magnitude greater in the RM than in the MFM. This is explained by the fact that cortical excitation and inhibition are occurring at the same time in the RM because both are phase-independent in the RM. Thus they tend to cancel each other, and for the net excitation and inhibition after cancellation to be of significant magnitudes, the original magnitudes must be large. In contrast, the MFM has cortical excitation and cortical inhibition occurring at different times because inhibition is antiphase from excitation. Thus when cortical excitation is large in magnitude, cortical inhibition will be small in magnitude, and vice versa. This relationship allows the cortical weights in the MFM to be much smaller for cortical connections to have a substantial effect on a cell's spiking output.

\section{The modified recurrent model}

We constructed a new model, the modified recurrent model (MRM), which essentially is the RM with antiphase inhibition of the MFM added. We started with the RM exactly like the one described above. We will call the excitatory cells $E$ cells and the inhibitory cells I cells. These cells have LGN input and phase-independent recurrent cortical connections exactly as described for the RM above. We then added a third set of V1 cells that are antiphase inhibitory cells. We will call these cells AI cells. The AI cells receive LGN input and cortical excitation from $E$ cells of the same spatial phase, using the correlation-based rule from the MFM with an Npow value of 6 . These cells then deliver inhibition to the $\mathrm{E}$ and I cells, again using the correlation-based rule and an Npow of 6 so that the maximum inhibition is to cells with opposite spatial phases. Our simulations with the MRM used 512 AI cells and 512 I cells (i.e., for each category of inhibitory cell, there was one cell of each orientation/phase type) as well as 512 E cells. The respective sums of all recurrent excitatory connection strengths, recurrent inhibitory connection strengths, and antiphase inhibitory connection strengths were each individually normalized to one. We set $e \rightarrow e$ and $e \rightarrow i$ to $3.2, i \rightarrow e$ and $i \rightarrow i$ to 3.5 , $e \rightarrow a i$ to $0.7, a i \rightarrow e$ and $a i \rightarrow i$ to 0.2 , and $F \rightarrow e, F \rightarrow i$, and $F \rightarrow$ ai to 0.07 . The background V1 activity resulting from LGN input in the absence of a stimulus was 0 spikes/s for E cells and I cells, and 5.7 spikes/s for AI cells.

\section{The inhibition-dominant recurrent model}

We also ran some simulations with the inhibition-dominant RM of McLaughlin et al. (2000). This model, like the standard RM, uses phase-insensitive intracortical connections. In the standard RM, the inhibitory profile is broader (i.e., covers a broader range of orientation differences) than the excitatory profile to generate a net Mexican-hat profile (see Intracortical circuitry for the $R M$ above). This is the opposite in the inhibition-dominant RM. However, in the inhibitiondominant RM, inhibitory cells are more broadly tuned than excitatory cells to produce an effective Mexican hat. The most important difference between the standard and inhibition-dominant RMs is that in the latter, inhibition is much stronger than excitation. We have included these main features in our implementation. We first took the inhibitory and excitatory connectivity profiles from our standard RM and switched them so that the inhibitory profile is narrower than the excitatory profile. We then used aspect ratios of 4.54 and 2.0 for the V1 excitatory and inhibitory cells, respectively, to generate sharply tuned excitatory cells and broadly tuned inhibitory cells. Finally, we set $e \rightarrow e$ to $0.05, e \rightarrow i$ was set to $0.1, i \rightarrow e$ was set to $0.5, i \rightarrow i$ was set to 0.4 , and $F \rightarrow e$ and $F \rightarrow i$ were set to 0.5 . Our implementation was quite simplified compared with McLaughlin et al.'s original model; for example, we neglected the variability in V1 orientation 
selectivity (Ringach et al. 2002). However, the model is sufficient for our purpose of studying the receptive-field structure of welltuned cells.

\section{Parametric variations}

Our conclusions in this paper are robust against the model parameters. For example, Fig. 3 shows that the RM cannot generate sharply tuned simple cells when the intracortical connection strengths vary in small steps from 0 to the full strengths. In addition, we reached the same conclusion when we doubled the sampling density of the LGN inputs, changed the contrast-response curves for our LGN responses, or used different Mexican-hat interaction profiles.

In our implementations of the two models, we followed the standard assumptions that the RM and MFM have a low (2.0) and high (4.54) aspect ratio for their LGN input connection pattern, respectively (Fig. 1, $A 1$ and $B 1$ ). We also ran some simulations of the RM with a 4.54 aspect ratio and some simulations of the MFM with a 2.0 aspect ratio. In the former case, the input was already well sharpened, and recurrent cortical connections simply removed the DC component to maintain robust contrast invariance. Interestingly, even in this case, the cells were complex, with response modulation ratios $(\mathrm{F} 1 / \mathrm{F} 0)<1$. In the latter case, the antiphase inhibition still removed the DC components to maintain the contrast invariance and keep cells simple. However, because there is no cortical sharpening of orientation tuning in the MFM, the model V1 cells' output firing was not well tuned for orientation. In either case, the conclusion that the MFM and the RM generate contrast-invariant simple and complex cell receptive-field structure remains the same.

\section{RE S U L T S}

We start by presenting our comparisons between the RM and MFM because the other models we considered depend on them. We will then show our simulations of the recent variations of the RM and MFM, i.e., the inhibition-dominant RM and the MFM with complex inhibition. Finally, we will present our MRM, which combines the key features of the RM and MFM.

\section{The multiphase RM and the MFM response to drifting gratings}

After implementing the multiphase MFM and the multiphase RM at the same level of complexity (see METHODS and Fig. 1), we compared the response properties of the two models. In particular, we wanted to know whether the cells in the two models behaved like simple or complex cells. Because the F1/F0 ratio calculated from a cell's response to a sinusoidal grating is widely used for classifying the cell as either simple or complex (Movshon et al. 1978a,c; Skottun et al. 1991), we first used the ratio to assess our model cells. The F0 component of a response time course is the unmodulated DC component, and the F1 component is the first harmonic (the component at the temporal frequency of the grating stimulus). The amplitude ratio of these two components is the $\mathrm{F} 1 / \mathrm{F} 0$ ratio, and a cell is classified as simple or complex according to whether the ratio is $>1$ or $<1$ (Skottun et al. 1991). Although the simple and complex cells may not form two discrete classes but are idealized cases of a continuum (Chance et al. 1999; Mechler and Ringach 2002; Tao et al. 2004), we can still use the ratio to indicate how simple or complex a cell is.

Each model contained V1 cells of different orientations and phases (as determined by the alignment of the LGN inputs). In the following, we will use the vertically oriented even-phase cell from each model as an example; cells of other orientations or phases have similar behavior. The LGN-to-V1 connectivity patterns for the two example cells are shown in Fig. 1, Al and $B 1$, respectively. We simulated their responses to a sinusoidal grating at the cells' preferred orientation and preferred spatial frequency, and a temporal frequency of $2 \mathrm{~Hz}$, which is the temporal frequency originally used to define the $\mathrm{F} 1 / \mathrm{F} 0$ ratio (Movshon et al. 1978a,c).

The results are shown in Fig. 2. For each model, we considered the special case with all intracortical connections set to zero (Fig. 2, first column), and the normal case with full-strength intracortical connections (Fig. 2, second column). The MFM and RM cells have similar response time courses when the intracortical connections are turned off (Fig. 2, Al and $B 1$ ). Both responses have large temporal modulations and the $\mathrm{F} 1 / \mathrm{F} 0$ ratio is 1.37 for both models, indicating that the cells are simple cells with phase sensitivity. However, intracortical connections are essential for both models to generate contrastinvariant orientation tuning. For the MFM, the intracortical connections did not reduce the temporal modulation of the response (Fig. 2A2). Instead, the antiphase inhibition in the MFM significantly suppressed the DC component of the responses, making the cell more simple with an F1/F0 ratio of 1.63. In contrast, the intracortical connections in the RM reduced the amplitude of the temporal modulation and increased the DC component of the response (Fig. 2B2), making the cell complex with an F1/F0 ratio of 0.38 . Note that simply establishing a threshold in Fig. $2 B 2$ to eliminate the DC component will not work. Such a threshold will eliminate the entire response to a low-contrast drifting grating.

Figure 2 suggests that the recurrent mechanism of the RM comes at a "cost" insofar as it cannot both sharpen orientation tuning and leave spatial phase information intact. To obtain a better sense of this relationship, we measured the relationship between the orientation tuning width and the F1/F0 ratio for both the MFM and the RM when the intracortical connections are varied systematically. The measure of tuning width is the standard half-width at half-height $(\mathrm{HWHH})$ of an orientation tuning curve. To vary the intracortical connections, we scaled the standard values (see METHODs) of all connection strengths by a factor between 0 and 1. The results are shown in Fig. 3, with the dashed and solid curves for the MFM cell and the RM cell, respectively. For both models, we began by setting all cortical connections to zero (as in Fig. 2, $A l$ and Bl). This represents the rightmost point for each curve in Fig. 3. As the scaling factor increases from 0 to 1 , the curves move toward the left, indicating sharper tuning (smaller HWHH). The leftmost points on the curves are for the full-strength intracortical connections. Note that, although both curves start at similar $\mathrm{F} 1 / \mathrm{F} 0$ ratios, they start at different $\mathrm{HWHH}$ values because the MFM has a feedforward input that is much more sharply tuned than the feedforward input in the RM. Indeed, the only reason that the cortical connections in the MFM change the HWHH value is that they eliminate the baseline DC component of the feedforward input (see Fig. 1). The tuned component of the feedforward input is not sharpened by the intracortical connections in the MFM.

In Fig. 3, the RM clearly shows a trade-off between the $\mathrm{F} 1 / \mathrm{F} 0$ ratio and tuning width as recurrent cortical connections are strengthened. There is no point on the curve where the 
A1

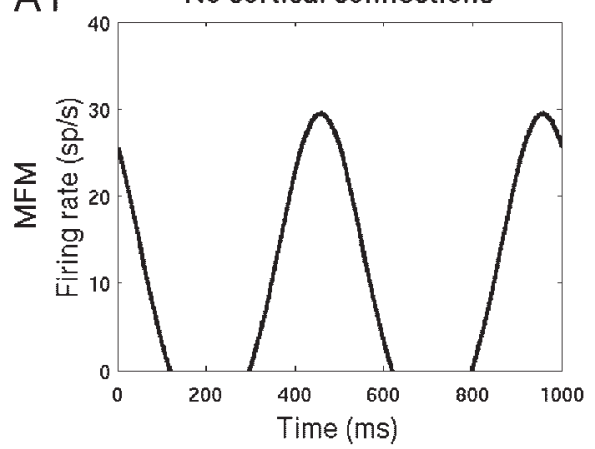

B1

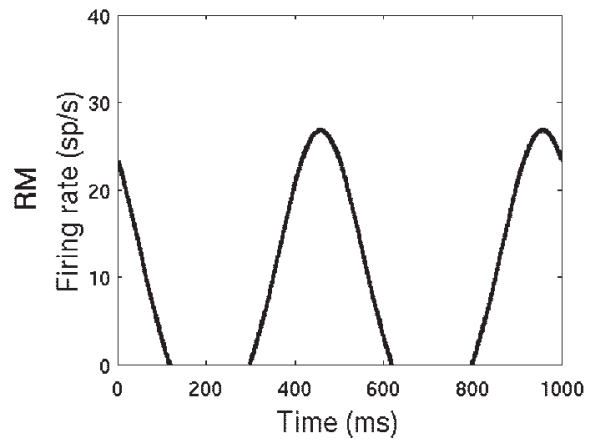

A2 Full-strength cortical connections

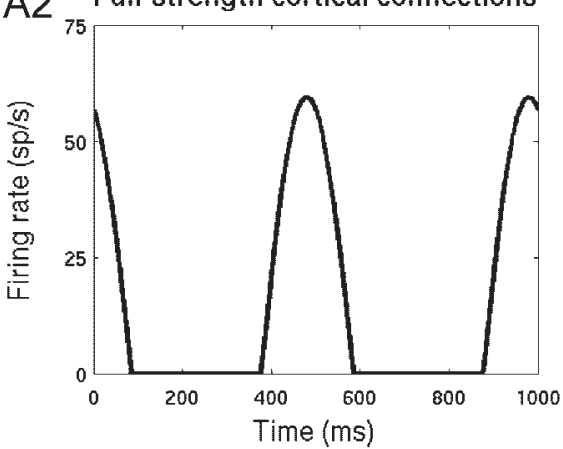

B2

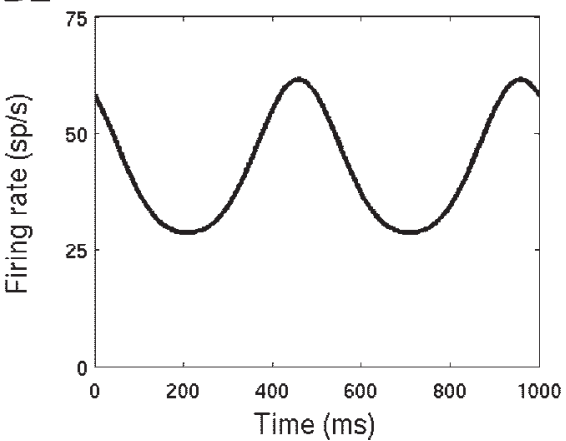

FIG. 2. Responses of a V1 cell from the MFM (top row) and a V1 cell from the RM (bottom row) to a 2-Hz drifting grating at the cells' preferred orientation (vertical) and preferred spatial frequency $(0.8$ cycles/deg). All panels show steadystate responses (after the initial transient response). $A 1$ and $B 1$ : temporal firing output of the model cells with the cortical connections turned off. $A 2$ and $B 2$ : temporal firing output of the model cells with the cortical connections turned on. Antiphase cortical inhibition in the MFM sharpens the cell's temporal modulation (A2), whereas the recurrent cortical connections in the RM increase the DC component (B2).
$\mathrm{F} 1 / \mathrm{F} 0$ ratio is $>1$ ( the physiological mean of about 20 to $25^{\circ}$. This confirms that the RM cannot both generate sharp orientation tuning and leave spatial phase information intact. By contrast, with increasing intracortical connections, the MFM shows the expected increase in the $\mathrm{F} 1 / \mathrm{F} 0$ ratio as the $\mathrm{HWHH}$ decreases. Therefore the RM can be a valid orientation tuning mechanism only for complex cells, whereas the MFM is suitable for simple cells. However, because the orientation tuning width of V1 cells

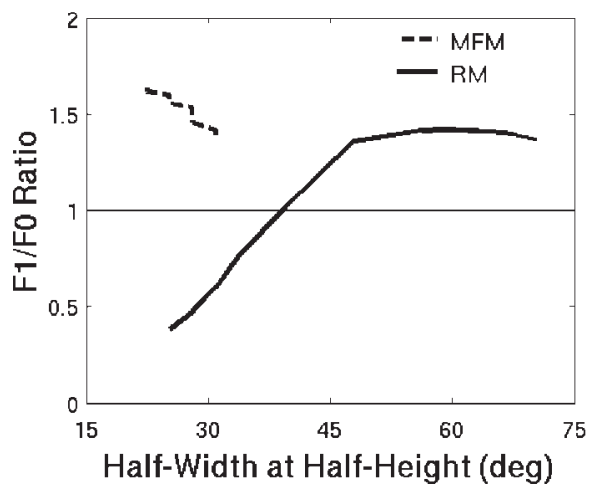

FIG. 3. Dependency of the response modulation ratio (F1/F0) and the tuning width on the intracortical connections in the MFM (dashed curve) and the RM (solid curve). Tuning width is measured by half-width at half-height (HWHH). For both models, we started with all cortical connections set to zero (the rightmost points of the curves), and then gradually increased the cortical connection strengths until they reached their full standard values (leftmost points on the curves). Note that both models have F1/F0 $>1$ (horizontal thin line) for a purely feedforward input (the rightmost point on each curve). As we increase the strength of the cortical connections, the F1/F0 ratio of the MFM increases, keeping the ratio $>1$. However, in the RM, the same procedure is accompanied by a decrease in the F1/F0 ratio, making the cell complex. Cortical connections reduce HWHH in both models. However, the feedforward input in the MFM is already well tuned for orientation; subsequent sharpening by the cortical connections mainly arises from elimination of the DC component. varies considerably, it is possible that the RM mechanism with weak recurrent connections is involved in some weakly orientation tuned simple cells, such as those in the input layer of monkey V1.

\section{The multiphase RM and MFM responses to bars}

Because Hubel and Wiesel (1962) used bar stimuli to define simple and complex cells, we confirmed our above conclusions with bars. Briefly, we simulated the responses of the two example V1 cells to either a vertical light bar or a vertical dark bar aligned with the on subregion of the receptive field for 400 ms. The time courses of the LGN inputs in the two models were very similar. However, the firing outputs from the two models are quite different. For the MFM cell, the light bar in the ON subregion generated an ON response but not an OFF response, and the dark bar in the on subregion generated an oFF response but not an on response, mirroring the LGN input to the cell. These are the characteristics of a simple cell. In contrast, the RM cell had both oN and ofF responses regardless of the type of bar, and thus behaved like a complex cell. In other words, for the RM cell, identities of the ON and OFF subregions determined by the LGN inputs are lost at the level of output firing.

\section{Single-phase $R M$ response to bars}

We also examined whether the introduction of some phase specificity into the recurrent connections of the RM would allow the cells in the model to preserve their simple-cell identity. In fact, the single-phase model of Somers et al. (1995) can be viewed as restricting the recurrent interactions in the $\mathrm{RM}$ to the cells with the same receptive field phase (but different orientations) only and, as we mentioned in the INTRODUCTION, Somers et al.'s model cells do have discrete on and 
OFF subregions. We have reproduced Somers et al.'s results by using our implementation of the single-phase RM described in METHODS. We found that indeed, the model cells retain their phase sensitivity (results not shown). However, the singlephase RM has a problem not noted previously: a model V1 cell's preferred orientation can change by $90^{\circ}$ depending on whether a light bar or a dark bar is used to measure the tuning. To illustrate this problem, we considered a single-phase RM consisting of even-phase V1 cells only, each with a central oN region flanked by two off regions according to the alignment of the LGN inputs. There are Mexican-hat recurrent connections among the cells with the same even phase but different orientations. We considered a vertically oriented example cell from the model and generated tuning curves from the on responses evoked by light and dark bars centered on the central on region of the receptive field.

Figure 4 shows the results, together with those from the corresponding multiphase RM cell for comparison. Figure $4 A$ shows the tuning curves of the feedforward inputs for the light and dark bars. They are identical for the single-phase and the multiphase RM cells. Figure $4, B$ and $C$ shows the tuning curves of the output firing for the single-phase RM cell and the multiphase RM cell, respectively. When a light bar is used to stimulate the single-phase RM, the cell prefers vertical orientation, as expected (Fig. 4B, solid curve). However, when a dark bar is used instead, the peak of tuning shifts by $90^{\circ}$ (Fig. $4 B$, dashed curve). This can be understood from the feedforward inputs in Fig. 4A: a light and a dark bar aligned with the central ON subregion generates a maximum and a minimum response, respectively, leading to a $90^{\circ}$ shift between the light and dark bars' feedforward tuning peaks. The single-phase RM simply amplifies around the peak of the feedforward tuning and suppresses other orientations, thus preserving this $90^{\circ}$ shift in the output firing. In contrast, the multiphase RM does not have this problem of peak shift (Fig. 4C) because of the strong excitation among cells with the same orientation but difference phases. For example, when the example cell receives minimal feedforward input generated by a dark bar aligned with its central on region, the cell with the opposite receptive field phase receives maximal feedforward input and sends strong excitation to the example cell to keep it activated as well.
Because real V1 cells do not show a $90^{\circ}$ peak shift according to the polarity of the bars (Hubel and Wiesel 1962, 1968), we conclude that the single-phase RM is not a viable model for simple cells. It should be noted, however, that Somers et al. (1995) designed the model to investigate primarily how recurrent cortical interactions shape feedforward inputs; they were not particularly concerned with receptive-field structures. It is thus reasonable for them to make the single-phase simplification. Indeed, every model has to use simplifying assumptions appropriate for the question under investigation that may not be appropriate for other questions.

\section{Alternative models of orientation tuning}

The above results show that the MFM and RM are well suited for explaining sharp orientation tuning in simple and complex cells, respectively. For completeness, we also simulated two variations of the MFM and the RM. They are the MFM with complex-cell inhibition (Lauritzen and Miller 2003) and the inhibition-dominant RM (McLaughlin et al. 2000; Tao et al. 2004; Wielaard et al. 2001). We constructed these models at the same level of detail as the MFM and the RM above, and found that they replicated simple cell physiology, as detailed below. When the excitation in the inhibition-dominant RM is substantially increased, it becomes similar to the standard RM and produces complex cells (Tao et al. 2004).

\section{The MFM with complex inhibition}

The MFM has recently been modified to include untuned complex-cell inhibition (Lauritzen and Miller 2003); this is in response to the finding that such inhibitory cells exist in layer 4 of the cat V1 (Hirsch et al. 2003), although their fraction in layer 4 and connectivity to other cells are not known. The same physiological study also found a couple of poorly tuned inhibitory simple cells in layer 4 that could provide antiphase inhibition in theory. Thus the new MFM has untuned complex inhibition in addition to antiphase inhibition. We found that the new MFM produced well-tuned simple cells just like the original MFM (Fig. 5). The only difference was that the untuned complex inhibition slightly reduced the role for an-
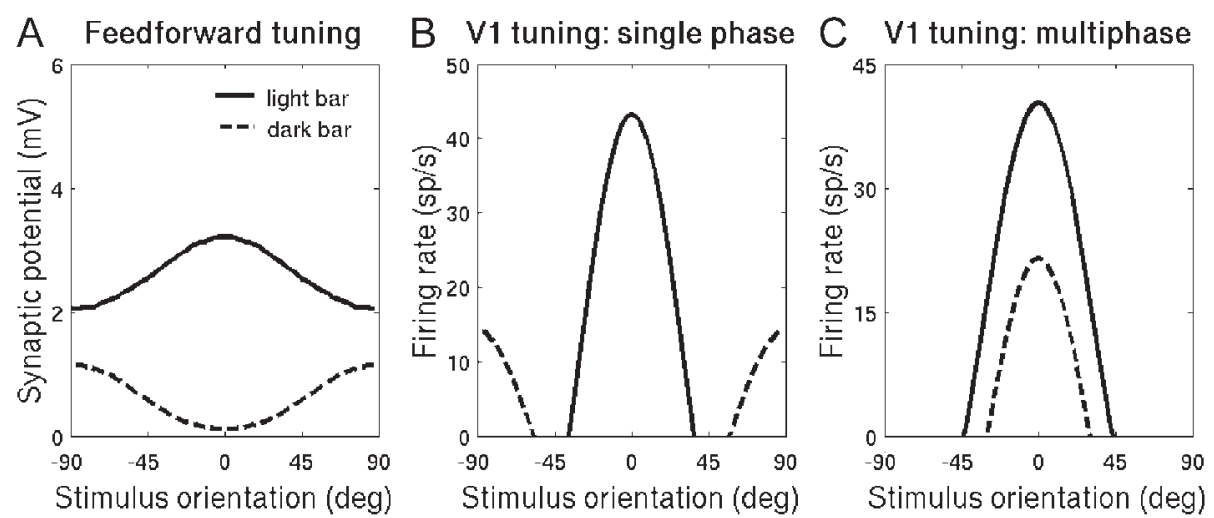

FIG. 4. Comparison between single-phase and multiphase RMs showing that the single-phase RM has the problem of producing orthogonal tuning peaks for light and dark bars. Example cells used here are vertically oriented and even phase as in the previous figures. Bars are centered on the central on region of the receptive field, and the tuning curves used here are made from the on responses $400 \mathrm{~ms}$ after stimulus onset. $A$ : tuning curves of the feedforward inputs, with the peak and the trough at the cell's preferred orientation for the light bar (solid curve) and dark bar (dashed curve), respectively. They are the same for the single-phase and multiphase RM cells. $B$ : tuning curves of the firing output for the single-phase RM cell. Problem is that they simply follow the input curves, with the tuning peaks differing by $90^{\circ}$ for the light and dark bars. $C$ : tuning curves of the firing output for the corresponding mulitiphase RM cell, with the same peak location for the light and dark bars. 


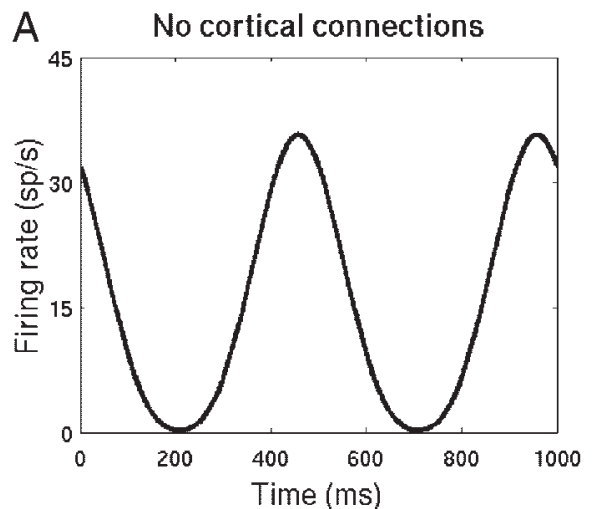

B Full-strength cortical connections

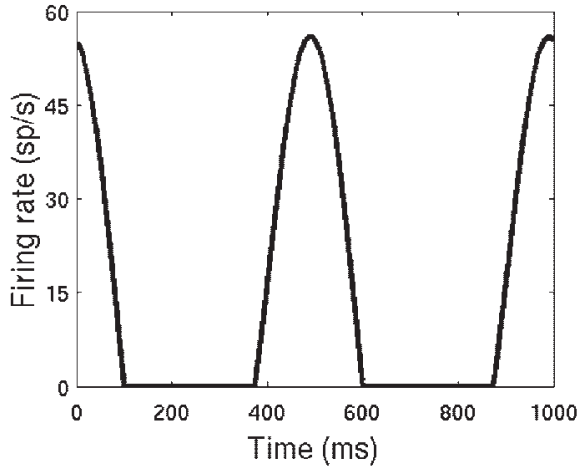

FIG. 5. Steady-state responses of a V1 cell from the MFM with complex inhibition to a $2-\mathrm{Hz}$ drifting grating at the cell's preferred orientation (vertical) and preferred spatial frequency $(0.8 \mathrm{cy}$ cles/deg). Temporal firing outputs of the cell with the cortical connections turned off and on are shown in $A$ and $B$, respectively. tiphase inhibition in eliminating the orthogonal response; now the two mechanisms shared this responsibility. One might predict that this would mean that the cortical connections would not increase the $\mathrm{F} 1 / \mathrm{F} 0$ ratio quite as much because the antiphase effect would be weaker. However, our simulations yielded an F1/F0 ratio of 1.61 when complex inhibition was added (similar to the 1.63 value with pure antiphase inhibition), probably because complex inhibition also serves to reduce the DC component in a cell's temporal response.

\section{The inhibition-dominant RM}

The inhibition-dominant RM of McLaughlin et al. (2000) is a version of the RM that includes detailed V1 anatomy. Interestingly, McLaughlin et al. implemented the Mexican-hat intracortical interactions not by a broader inhibitory connectivity profile but by broader tuning of inhibitory cells (see METHODS). Because the complex-cell behavior in any RM stems from strong net recurrent excitation (the peak of the Mexican hat) among cells with different phases, one expects that if the recurrent inhibition is much stronger than recurrent excitation (and thus removes the peak of the Mexican hat), the resulting inhibition-dominant RM should produce simple cells. This is indeed what McLaughlin et al. found (McLaughlin et al. 2000; Wielaard et al. 2001). We have reproduced this finding in Fig. 6.

The inhibition-dominant RM makes the strong prediction that the net effect of cortical connections on simple cells is inhibitory. Specifically, the cortex inhibits a simple cell's response to LGN input at every orientation (including the preferred orientation). This is why in Fig. 6, the LGN input to the cell is larger than it is in the other models. In contrast, the
$\mathrm{RM}$, the MFM, and the MRM presented below all predict that cortex suppresses the LGN input only away from a V1 cell's preferred orientation, but boosts the LGN input around the cell's preferred orientation. (The MFM boosts responses at the preferred orientation through phase-specific cortical excitation. Because the recurrent excitation in the inhibition-dominant RM is not phase specific, any such boosting leads to complex cell behavior as in the original RM.) Wielaard et al. (2001) examined the inhibition-dominant RM further and found that when cortical connections are silenced in the model, a simple cell's response at its preferred orientation increases by threefold. Cortical inactivation experiments in cat V1 (Chung and Ferster 1998; Ferster et al. 1996) found a decrease, although similar experiments have not been done in monkeys (see DISCUSSION).

\section{The modified recurrent model (MRM)}

Because the antiphase connections in the MFM were found to increase the F1/F0 ratio (Fig. 3), we asked whether antiphase inhibition could make a recurrent mechanism relevant for sharp orientation tuning in simple cells. To do this, we started with a multiphase RM with phase-independent intracortical connections. We then introduced an additional set of inhibitory cells that received feedforward input from the LGN and same-phase excitatory connections from cortical excitatory cells in the RM. This extra set of inhibitory cells supplies antiphase inhibition to all cortical cells in the RM (see METHODS). What resulted was a phase-dependent response well tuned for orientation. Both the ON and OFF response to light and dark bars (results not shown) and the modulated response to a drifting grating (Fig. 7) showed spatial phase dependency, with the latter yielding an F1/F0 ratio of 1.53 . This demonstrates that phase-independent
A

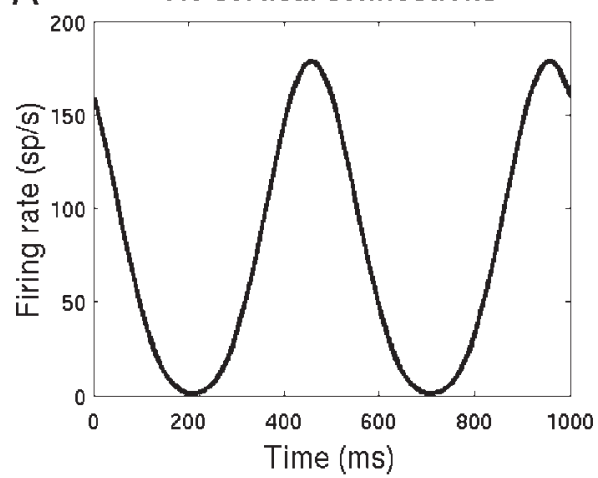

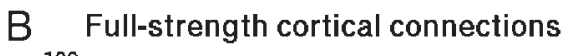

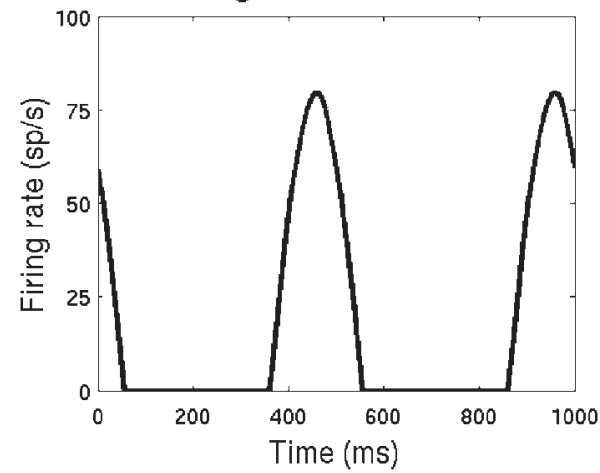

FIG. 6. Steady-state responses of a V1 cell from the inhibition-dominant RM to a $2-\mathrm{Hz}$ drifting grating at the cell's preferred orientation (vertical) and preferred spatial frequency $(0.8$ cycles/deg). Temporal firing outputs of the cell with the cortical connections turned off and on are shown in $A$ and $B$, respectively. 

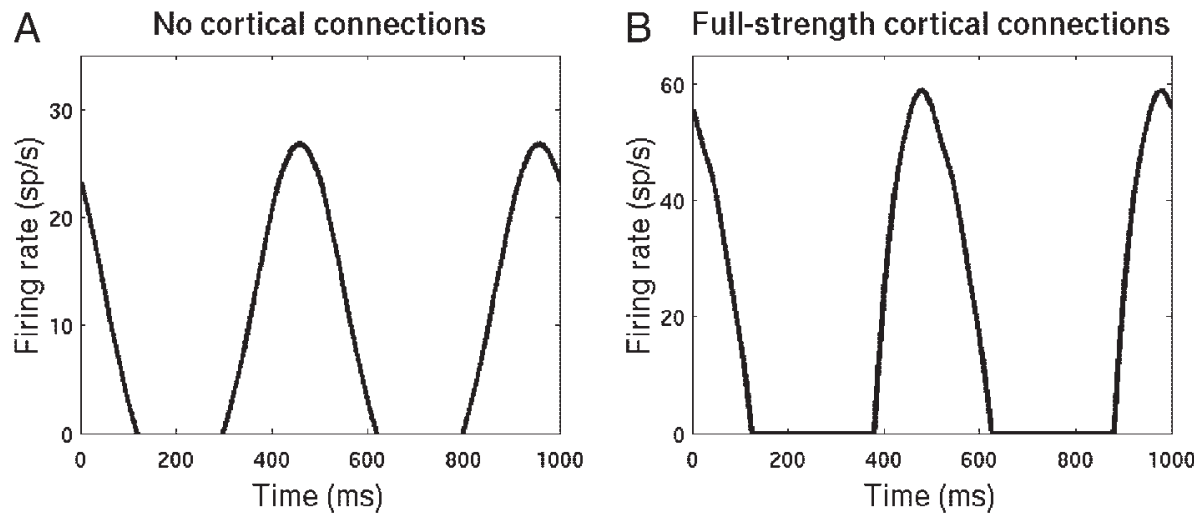

FIG. 7. Steady-state responses of a V1 cell from the MRM to a $2-\mathrm{Hz}$ drifting grating at the cell's preferred orientation (vertical) and preferred spatial frequency $(0.8$ cycles/deg). Temporal firing outputs of the cell with the cortical connections turned off and on are shown in $A$ and $B$, respectively. recurrent connections and antiphase inhibition together can provide a mechanism for orientation tuning in V1 simple cells. In this modified recurrent model (MRM), the phase-independent recurrent connections sharpen orientation tuning and the antiphase inhibition preserves phase dependency. The cell in Fig. 7 had an $\mathrm{HWHH}$ value of around $25^{\circ}$.

By adding antiphase inhibition to the RM, we not only produced well-tuned simple cells, but also created a model whereby a cell can have a range of F1/F0 values, from simple to complex, by varying the relative strengths of antiphase and recurrent inhibition. As noted in METHODS, the MRM has three types of cortical cells: excitatory (E), inhibitory (I), and antiphase inhibitory (AI). The E and I cells make up the Mexicanhat interaction profile that sharpens tuning bandwidths and the AI cells primarily enforce phase specificity. We started with the MRM parameters used above that generated well-tuned simple cells (the topmost point of the curve in Fig. 8). We then gradually reduced the AI connection strength from 0.2 to 0 and at the same time increased the I connection strength from 3.5 to 3.9. The fractional decrease of the AI connections was matched by the fractional increase of the I connections to keep tuning curve amplitudes roughly consistent. At the end of the process, the cells were complex (the lowest point in Fig. 8) because this model is essentially the RM. The intermediate cases cover a broad range of the $\mathrm{F} 1 / \mathrm{F} 0$ ratios or varying degrees of simple-complex behavior, all with sharp orientation tuning (Fig. 8). This result of the MRM should be compared

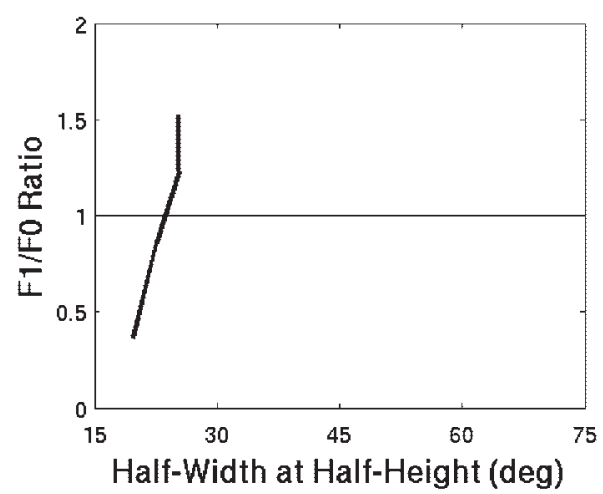

FIG. 8. MRM can produce well-tuned cells with varying degrees of simplecomplex behavior by varying the relative strengths of antiphase and recurrent inhibition. MRM with strong antiphase inhibition (used in Fig. 7 for simple cells) corresponds to the topmost point on the curve, with F1/F0 ratio $>1$. MRM with no antiphase inhibition (equivalent to the RM) corresponds to the lowest point on the curve. Intermediate combinations of antiphase and recurrent inhibition produced a full spectrum of $\mathrm{F} 1 / \mathrm{F} 0$ values. with that for the MFM and the RM in Fig. 3. The RM is unable to produce well-tuned simple cells; it can produce only welltuned complex cells. The MFM can produce well-tuned simple cells but not complex cells. Because real orientation-tuned V1 cells vary in their degree of simple-complex behavior, the MRM is the best model in this sense.

\section{I S C U S S I O N}

In this study, we implemented the MFM, the RM, and some of their variations at the same level of detail, and compared their receptive field properties. We found that over a broad range of cortical connection strengths, the RM cannot generate both sharp, contrast-invariant orientation tuning and strong phase dependency at the same time. Thus the standard RM is not suitable for explaining the emergence of sharp orientation tuning in V1 simple cells. In contrast, the MFM, with or without broad complex-cell inhibition, can generate both strong orientation tuning and phase dependency and behave like well-tuned simple cells. We also found that one cannot endow the RM with simple-cell properties by restricting the recurrent cortical connections to cells with the same receptive field phase (but different orientations); although such a singlephase RM is phase sensitive (Somers et al. 1995), the tuning peak can shift by $90^{\circ}$ depending on whether the stimulus is a light or a dark bar. An inhibition-dominant version of the RM produces simple cells (and also complex cells if the recurrent excitation is substantially increased), but it predicts that a simple cell's response to its preferred orientation should increase after cortical inactivation (see following text). Finally, we found that when the antiphase inhibition of the MFM is added to the multiphase RM, the resulting MRM does behave like real simple cells, with the recurrent mechanism sharpening the tuning and the antiphase inhibition maintaining the receptive field phase specified by the LGN inputs. By varying the relative strengths of the antiphase and recurrent inhibition, the MRM can produce well-tuned cells covering a broad range of simple to complex behaviors. We conclude that the standard MFM and RM are best suited to account for strong orientation selectivity in simple and complex cells, respectively. Although complex cells could also be constructed from simple cells with the MFM mechanism, the RM mechanism is needed to explain the plastic properties of orientation tuning (Felsen et al. 2002; Teich and Qian 2003; Teich and Qian, unpublished results). Because simple and complex cells may represent idealized cases of a continuum (Chance et al. 1999; Mechler and Ringach 2002; Tao et al. 2004), it is conceivable that both the 
RM and the MFM mechanisms are used to various degrees in V1, as suggested by our MRM simulations.

\section{Reconciling the RM and some data}

The assignment of the MFM and the RM to simple and complex cells helps avoid some apparent contradictions between the models and the experimental data. As we mentioned in the INTRODUCTION, two pieces of data have been noted as major evidence against the RM (Ferster and Miller 2000). First, when intracortical contributions are diminished by cortical inactivation, the intracellularly recorded orientation tuning curves do not become broader. The RM predicts broadening. Second, Vidyasagar and Siguenza (1985) found that the orientation tuning width decreases with increasing spatial frequency of the stimulus. The RM predicts that the tuning width should be largely determined by the Mexican-hat intracortical interaction profile and should only weakly depend on the feedforward tuning width. Both contradictions can be avoided if we assume the RM is just for complex cells. Ferster et al. recorded only input layer simple cells from cat V1 in their inactivation experiments (Chung and Ferster 1998; Ferster et al. 1996). Likewise, Vidyasagar and Siguenza (1985) indicated that the spatial-frequency dependency of orientation tuning width is significant only for simple cells but not for complex cells. Consistent with the results of Vidyasagar and Siguenza (and with the RM), Mazer et al. (2002) failed to find significant spatial-frequency dependency of orientation tuning in monkey V1 where most cells are complex.

\section{Plasticity properties of orientation tuning}

It has been shown that orientation adaptation and learning can alter the peak location and slope of orientation tuning curves of V1 cells (Dragoi et al. 2000; Felsen et al. 2002; Schoups et al. 2001; Teich and Qian 2003). Although this paper does not concern plasticity, we briefly discuss the issue here because of its relevance to evaluating models of orientation selectivity. Our previous work (Schoups et al. 2001; Teich and Qian 2003) and ongoing research (Teich and Qian, unpublished observations) indicate that to explain the observed plastic changes of orientation tuning, particularly those induced by adaptation, one has to assume a broad feedforward tuning and the Mexican-hat recurrent intracortical connections. Thus the MFM and the MFM with complex inhibition cannot explain the observed plastic properties whereas the RM and the MRM can. However, the multiphase RM model presented in this paper can become unstable when some specific cortical connections are dramatically reduced to mimic the effects of adaptation. This problem can be avoided if the RM cells achieve their complex cell status by mixing different phases of the feedforward inputs instead of by the recurrent inputs. The recurrent connections in such a phase-independent RM can then be altered to account for the observed plastic properties without any instability. In fact, the simplified RM used in our previous plasticity study (Teich and Qian 2003) is equivalent to such a phase-independent RM. The MRM presented herein does not have the stability problem of the multiphase RM because the antiphase inhibition makes the model stable during connection modifications. In summary, the MFM behaves like V1 simple cells but cannot explain the observed plastic prop- erties of orientation tuning. The MRM covers a spectrum of simple-complex behavior and can explain the plastic properties. The multiphase RM behaves like V1 complex cells but may become unstable when a specific pattern of large connection changes is introduced to model plastic properties. The phase-independent RM (through mixed-phase feedforward inputs) behaves like complex cells and can explain the plastic properties. The details of our modeling of orientation plasticity will be presented in another publication.

Series et al. (2004), who recently compared the RM and the MFM from the perspective of coding efficiency, suggested that learning-induced sharpening of orientation tuning curves in the RM (Teich and Qian 2003) cannot explain the postlearning improvement of orientation discriminability. Their main argument is that recurrent connections in the RM lead to correlations among cells' firing and a lower coding efficiency. However, in our simulations (Teich and Qian 2003), the sharpening was achieved by a reduction (instead of an increase) of the recurrent connections in the RM, which should reduce (not increase) the correlations between the cells, compared with the baseline RM before learning. Therefore the postlearning improvement caused by the sharpening of tuning in the RM may be even stronger when the correlations between the cells' firing are considered. Series et al. showed that based on the coding efficiency alone, the brain should use a feedforward architecture to create new tuning. However, they did not show how the observed orientation plasticity might be accounted for by the feedforward model.

\section{The models of McLaughlin et al. and the inhibition- dominant RM}

McLaughlin et al. recently proposed large-scale models of V1 orientation selectivity based on more detailed anatomy of monkey V1 (McLaughlin et al. 2000; Wielaard et al. 2001). Although their early implementations consider only a single receptive field phase according to the connectivity of the feedforward inputs (McLaughlin et al. 2000; Wielaard et al. 2001), those models are effectively multiphase models because of the random variations of the spatial location of receptive fields. Their later implementation directly includes multiple receptive field phases (Tao et al. 2004). We group their models with the RM because they also use phase-independent Mexican-hat intracortical interactions, although the Mexican hat is realized through different tuning widths between inhibitory and excitatory cells, instead of through different connectivity ranges in the standard RM (see METHODS). When the net recurrent excitation of the Mexican hat is strong in McLaughlin et al.'s models, the cells behave like complex cells (Tao et al. 2004), just as in the standard RM. On the other hand, when the net recurrent excitation is largely eliminated, the resulting inhibition-dominant RM generates simple cells (Tao et al. 2004), as reproduced in our Fig. 6. By varying the relative strengths of recurrent excitation and inhibition, cells with different degrees of simple-complex behavior can be produced (Tao et al. 2004).

As mentioned earlier in RESULTS, the inhibition-dominant RM makes the critical prediction that, for V1 simple cells, net intracortical interactions are inhibitory across all stimulus orientations; consequently, a simple cell's response at its preferred orientation should increase after cortical inactivation 
(Wielaard et al. 2001). In contrast, the RM, the MFM, and the MRM all assume that there is cortical boosting of responses at a cell's preferred orientation and cortical inactivation should reduce cells' preferred responses. Ferster and coworkers found large response reductions $(50-65 \%)$ at the preferred orientation of cat simple cells after cortical inactivation (Chung and Ferster 1998; Ferster et al. 1996). Note that although Ferster et al. reported only the F1 component of the cell's responses, the predictions discussed here hold for the F1 component. However, similar inactivation experiments have not been done in monkey V1 modeled by McLaughlin et al. Further experiments are needed to resolve this issue.

\section{Mechanisms of Vl orientation tuning}

The main goal of comparing the MFM, the RM, and their variants is, of course, to determine which mechanism is more relevant to the emergence of strong orientation tuning in V1. We have already touched on this issue above and will attempt a more comprehensive discussion here. We first note that certain properties of $\mathrm{V} 1$ orientation tuning can be explained by either model. These include the contrast invariance and the inhibition blockade experiments (Somers et al. 1995; Troyer et al. 1998). The RM can additionally explain the preserved orientation tuning under the blockade of the on channel (Somers et al. 1995). Because the MFM has not been applied to this finding, we simulated the blockade of the on-center inputs to V1 in our implementation of the MFM and found that the MFM is also consistent with these findings (results not shown); the primary effect, consistent with the data (Horton and Sherk 1984; Schiller 1982; Sherk and Horton 1984), was an amplitude reduction. There was no broadening of tuning.

We have considered other relevant experimental results that may discriminate the two models, and in some cases, we have reached different conclusions from what are commonly assumed in the literature. The input layer of cat V1 is dominated by simple cells (Hubel and Wiesel 1962). Because we have shown in this paper that the RM cells are not simple, the RM must not be responsible for the initial emergence of strong orientation tuning in cat V1. The MFM and the MRM are more viable candidates. However, it is possible for the standard RM mechanism to be involved in further refinements of orientation tuning at the complex-cell level outside the input layer. In addition, most input layer cells of monkey V1 are, like LGN cells, not well tuned to orientation, and the majority of the cells outside the input layer are complex. Thus the RM may well be responsible for the emergence of strong orientation selectivity in monkey complex cells. Both the inhibition-dominant RM and the MRM can produce a continuum of simple- to complexcell behavior with sharp orientation tuning. However, the former is not a viable model for cat V1 (and was not designed for cat V1) because it is inconsistent with Ferster et al.'s finding of reduced simple-cell response at the preferred orientation under cortical inactivation. Whether the model is applicable to monkey V1 depends on whether Ferster et al.'s finding can be replicated in monkey.

As we mentioned above, the cortical inactivation experiments were done with simple cells and the spatial-frequency dependency of orientation tuning was found in simple, but not complex, cells. These data further rule out the role of the RM in orientation selectivity of V1 simple cells. By restricting the
RM to complex cells, the apparent contradiction between these data and the RM can be avoided. The cortical inactivation experiments provided a main motivation for Troyer et al. (1998) to propose the MFM as an alternative to the RM. But how well is the MFM supported by the cortical inactivation experiments? Recall that the MFM relies on feedforward inhibition to maintain contrast invariance. This inhibition (whether antiphase or untuned complex) can be provided only by V1 interneurons. Cortical inactivation by cooling or electrical shock should thus diminish the inhibition and destroy contrast invariance. This means that if $\mathrm{HWHH}$ is used to measure tuning width, the MFM predicts that under high stimulus contrast, the intracellularly recorded tuning curves should be broader under the cortical inactivation condition than under the normal condition. More specifically, the inactivation-generated broadening should be mainly the result of a contrast-dependent, orientation-untuned DC component that, under the normal condition, is suppressed by the antiphase inhibition (Shapley et al. 2003; Sompolinsky and Shapley 1997). Unfortunately, Ferster et al. reported only the F1, but not the DC, component of the intracellular responses. In addition, they did not test contrast invariance under the inactivation condition. A critical test of the MFM would be to measure the contrast invariance and the DC component of tuning under the cortical inactivation condition.

A controversy related to the mechanism of orientation selectivity is whether orientation tuning curves change their width or shape over time (Celebrini et al. 1993; Gillespie et al. 2001; Mazer et al. 2002; Pei et al. 1994; Ringach et al. 1997, 2003; Shapley et al. 2003; Sharon and Grinvald 2002; Shevelev et al. 1993). A common assumption is that if orientation tuning curves of V1 cells are fully sharpened immediately after a stimulus is presented, then the feedforward input must be sharply tuned. On the other hand, if tuning curves are initially broad and sharpen over time, then the recurrent cortical connections must play a role in the sharpening of orientation tuning curves. However, because the strict feedforward model of Hubel and Wiesel is known to be incomplete as a result of its lack of contrast invariance, one must use the MFM as a viable version of the feedforward model. When the tuning width is measured by HWHH (as is usually the case), even the MFM predicts a cortical sharpening of the tuning curve over time because the antiphase inhibition in the MFM removes the untuned DC component in the feedforward inputs. Thus depending on how the tuning width is measured, cortical connections can significantly change the width of tuning curves over time in both models. Because the temporal evolution of tuning sharpening can be very fast in a model (Somers et al. 1995), it may be difficult to distinguish the models based on the temporal dynamics measurements.

Another point of contention in the recurrent/feedforward debate concerns the nature of inhibition. Note that all models showing contrast-invariant orientation tuning require net inhibition at off-optimal orientations to suppress the contrastdependent DC responses (Shapley et al. 2003; Sompolinsky and Shapley 1997). In the MFM, this inhibition is provided by broadly tuned simple cells (with the strongest inhibition between antiphase pairs) and untuned complex cells; in the RM, it is provided by a broad connectivity profile of inhibitory complex cells whose tuning widths are not important; and in the inhibition-dominant RM, it is provided by broadly tuned 
inhibitory simple cells with phase-independent connectivity. As we mentioned in METHODS, there is evidence for inhibition at a cell's nonpreferred orientations including the orthogonal orientation (Allison et al. 2001; Bonds 1989; Martinez et al. 2002; Monier et al. 2003; Shapley et al. 2003). More studies on the fractions of inhibitory cells of various physiological properties and their connectivity to other cells are obviously needed for differentiating the models.

Carandini and Ringach (1997) considered how the RM responds when the stimulus contains two different orientations. They found that for two orientations differing by as much as $30^{\circ}$ in the stimulus, the population response has only one peak. This result might be viewed as evidence against the RM because the model fails to produce two peaks under the condition where we easily perceive two orientations. However, it has been shown in the case of motion direction that real MT cells have only one peak in their response to composite motion stimuli that generate a perception of two motion directions (Treue et al. 2000). Thus the number of perceived orientations may not correspond to the number of peaks in neural responses. Besides, it is unlikely that the MFM responses will always have two peaks for stimuli that generate a perception of two orientations.

We have mentioned above that the RM (or the more general MRM) is much better suited for explaining the observed plastic properties of orientation tuning than the MFM with or without complex inhibition. Because not all V1 cells show significant orientation plasticity, we cannot rule out the MFM as a mechanism for V1 orientation tuning. However, we can at least say that the MFM by itself is insufficient to explain all the observed properties of V1 orientation selectivity.

Our discussions above suggest that overall, the MRM, which combines the main features of the RM and MFM, appears to be the most general model for describing V1 orientation tuning. The MRM is not only consistent with orientation plasticity data, but can also explain the simple- to complex-cell continuum all well tuned to orientation. Furthermore, the MRM is consistent with experiments showing that the net effect of

TABLE 1. Summary of main features of the different models

\begin{tabular}{|c|c|c|c|c|c|c|}
\hline & $\begin{array}{l}\text { Hubel-Wiesel } \\
\text { Feedforward } \\
\text { Model }\end{array}$ & $\begin{array}{l}\text { Recurrent Model } \\
(\mathrm{RM})\end{array}$ & $\begin{array}{c}\text { Modified } \\
\text { Feedforward } \\
\text { Model }(\mathrm{MFM})^{\mathrm{a}}\end{array}$ & $\begin{array}{c}\text { Inhibition- } \\
\text { Dominant RM }\end{array}$ & $\begin{array}{l}\text { MFM With } \\
\text { Complex } \\
\text { Inhibition }\end{array}$ & $\begin{array}{c}\text { Modified } \\
\text { Recurrent Model } \\
\text { (MRM) }\end{array}$ \\
\hline $\begin{array}{l}\text { Subregion aspect } \\
\text { ratio of } \\
\text { feedforward input }\end{array}$ & Large & Small & Large & $\begin{array}{l}\text { A range }{ }^{\mathrm{b}} \text { from } \\
\text { small to large }\end{array}$ & Large & Small \\
\hline $\begin{array}{l}\text { Tuned component } \\
\text { of feedforward } \\
\text { input }\end{array}$ & Sharp & Broad & Sharp & $\begin{array}{l}\text { A range from } \\
\text { broad to sharp }\end{array}$ & Sharp & Broad \\
\hline Cortical excitation & None & Phase-independent & Phase-dependent & Phase-independent & Phase-dependent & Phase-independent \\
\hline Cortical inhibition & None & Phase-independent & Phase-dependent & Phase-independent & $\begin{array}{l}\text { Both phase- } \\
\text { dependent and } \\
\text {-independent }\end{array}$ & $\begin{array}{l}\text { Both phase- } \\
\text { dependent and } \\
\text {-independent }\end{array}$ \\
\hline $\begin{array}{l}\text { Net inhibition at } \\
\text { nonoptimal } \\
\text { orientations }\end{array}$ & None & $\begin{array}{l}\text { Yes, through } \\
\text { broader range } \\
\text { of inhibitory } \\
\text { connections }\end{array}$ & $\begin{array}{l}\text { Yes, through DC } \\
\text { component of } \\
\text { inhibitory cells }\end{array}$ & $\begin{array}{l}\text { Yes, through } \\
\text { broader tuning } \\
\text { of inhibitory } \\
\text { cells }\end{array}$ & $\begin{array}{l}\text { Yes, through DC } \\
\text { component of } \\
\text { simple and } \\
\text { broader tuning } \\
\text { of complex } \\
\text { inhibitory cells }\end{array}$ & $\begin{array}{l}\text { Yes, through } \\
\text { broader range } \\
\text { of inhibitory } \\
\text { connections }\end{array}$ \\
\hline $\begin{array}{l}\text { Intracortical } \\
\text { sharpening of } \\
\text { tuning }\end{array}$ & No & Yes & $\begin{array}{l}\text { Yes overall, no } \\
\text { for the tuned } \\
\text { component }\end{array}$ & Yes & $\begin{array}{l}\text { Yes overall no for } \\
\text { the tuned } \\
\text { component }\end{array}$ & Yes \\
\hline $\begin{array}{l}\text { Contrast invariance } \\
\text { of tuning }\end{array}$ & No & Yes & Yes & Yes & Yes & Yes \\
\hline $\begin{array}{l}\text { Cortical inactivation } \\
\text { destroys contrast } \\
\text { invariance }\end{array}$ & N/A & Yes & Yes & Yes & Yes & Yes \\
\hline $\begin{array}{l}\text { Cortical inactivation } \\
\text { broadens tuned } \\
\text { components }\end{array}$ & No & Yes & No & $\begin{array}{l}\text { Yes and no } \\
\text { depending on a } \\
\text { cell's aspect } \\
\text { ratio }\end{array}$ & No & Yes \\
\hline $\begin{array}{l}\text { Effect of cortical } \\
\text { inactivation at } \\
\text { preferred } \\
\text { orientation }\end{array}$ & None & $\begin{array}{l}\text { Decreased } \\
\text { response }\end{array}$ & $\begin{array}{l}\text { Decreased } \\
\text { response }\end{array}$ & $\begin{array}{l}\text { Increased response } \\
\text { for simple cells }\end{array}$ & $\begin{array}{l}\text { Decreased } \\
\text { response }\end{array}$ & $\begin{array}{l}\text { Decreased } \\
\text { response }\end{array}$ \\
\hline Receptive-field type & Simple & Complex & Simple & $\begin{array}{l}\text { Simple to } \\
\text { complex }\end{array}$ & Simple & $\begin{array}{l}\text { Simple to } \\
\text { complex }\end{array}$ \\
\hline $\begin{array}{l}\text { Plasticity of tuning } \\
\text { as observed }^{\mathrm{d}}\end{array}$ & No & $\begin{array}{l}\text { Yes, but with } \\
\text { some } \\
\text { instability }\end{array}$ & No & $\begin{array}{l}\text { Yes for cells } \\
\text { similar to those } \\
\text { in the RM, no } \\
\text { for other cells }\end{array}$ & No & Yes \\
\hline
\end{tabular}

\footnotetext{
${ }^{\mathrm{a}}$ A detailed comparison between the RM and the MFM can be found in the subsection Differences between the MFM and the RM in METHODS. ${ }^{\mathrm{b}}$ Obviously, other models could also incorporate a range of aspect ratios to increase richness of properties. ${ }^{\mathrm{c}} \mathrm{A}$ definition of the tuned component can be found in the subsection Differences between the MFM and the RM in METHODS. ${ }^{\mathrm{d}}$ Based on Teich and Qian (2003) and unpublished results. ${ }^{\mathrm{e}}$ We are considering the multiphase RM in this column because the single-phase RM has an incorrect peak shift shown in Fig. 4B. The instability occurs only when large changes to connections are introduced to model plasticity. The no-phase RM used by Teich and Qian (2003) does not have instability.
} 
cortical interactions in cats is to boost simple-cell responses to stimuli at the preferred orientation.

A summary of the main features of the different models discussed in this paper is presented in Table 1.

Our discussion also suggests that in the recurrent/feedforward debate, it is important to distinguish between different cells types and species and avoid overgeneralization from one cell type or species to another before the relevant data become available. It is also important to know the exact predictions of the models and exact scope of the available data before reaching a conclusion. Only through such careful comparisons can we identify those experiments that can best discriminate the models in the future.

\section{A C K N O W LED G MENT S}

We thank Drs. A. Das, M. Goldberg, J. Gottlieb, K. Miller, and R. Shapley and anonymous reviewers for many helpful discussions and comments. In addition, we are very grateful to Dr. Miller for providing a great deal of help in building our version of the modified feedforward model. We also used some code from Drs. M. Carandini and D. Ringach's version of the recurrent model, and thank them for making source code available.

\section{G R A N T S}

This work was supported by National Eye Institute Grants R01 EY-016270 (formerly MH-54125) and T32 EY-13933.

\section{REFERENCES}

Ahmed B, Anderson JC, Douglas RJ, Martin KA, and Nelson JC. Polyneuronal innervation of spiny stellate neurons in cat visual cortex. J Comp Neurol 341: 39-49, 1994.

Allison JD, Smith KR, and Bonds AB. Temporal-frequency tuning of cross-orientation suppression in the cat striate cortex. Vis Neurosci 18: 941-948, 2001.

Anderson JS, Carandini M, and Ferster D. Orientation tuning of input conductance, excitation, and inhibition in cat primary visual cortex. J Neurophysiol 84: 909-926, 2000b.

Anderson JS, Lampl I, Gillespie DC, and Ferster D. The contribution of noise to contrast invariance of orientation tuning in cat visual cortex. Science 290: 1968-1972, 2000a.

Ben-Yishai R, Bar-Or RL, and Sompolinsky H. Theory of orientation tuning in visual cortex. Proc Natl Acad Sci USA 92: 3844-3848, 1995.

Bonds AB. Role of inhibition in the specification of orientation selectivity of cells in the cat striate cortex. Vis Neurosci 2: 41-55, 1989.

Carandini M and Ringach DL. Predictions of a recurrent model of orientation selectivity. Vision Res 37: 3061-3071, 1997.

Celebrini S, Thorpe S, Trotter Y, and Imbert M. Dynamics of orientation coding in area V1 of the awake primate. Vis Neurosci 10: 811-825, 1993.

Chance FS, Nelson SB, and Abbott LF. Complex cells as cortically amplified simple cells. Nat Neurosci 2: 277-282, 1999.

Chapman B, Zahs KR, and Stryker MP. Relation of cortical cell orientation selectivity to alignment of receptive fields of the geniculocortical afferents that arborize within a single orientation column in ferret visual cortex. J Neurosci 11: 1347-1358, 1991.

Chen Y, Wang Y, and Qian N. Modeling V1 disparity tuning to time-varying stimuli. J Neurophysiol 86: 143-155, 2001.

Chung $\mathbf{S}$ and Ferster D. Strength and orientation tuning of the thalamic input to simple cells revealed by electrically evoked cortical suppression. Neuron 20: 1177-1189, 1998.

Douglas RJ, Koch C, Mahowald M, Martin KA, and Suarez HH. Recurrent excitation in neocortical circuits. Science 269: 981-985, 1995.

Douglas RJ, and Martin KA. Neuronal circuits of the neocortex. Аnпи Rev Neurosci 27: 419-451, 2004.

Dragoi V, Sharma J, Miller EK, and Sur M. Dynamics of neuronal sensitivity in visual cortex and local feature discrimination. Nat Neurosci 5 : 883-891, 2002.

Dragoi V, Sharma J, and Sur M. Adaptation-induced plasticity of orientation tuning in adult visual cortex. Neuron 28: 287-298, 2000.

Felsen G, Shen YS, Yao H, Spor G, Li C, and Dan Y. Dynamic modification of cortical orientation tuning mediated by recurrent connections. Neuron 36: 945-954, 2002.
Ferster D. Orientation selectivity of synaptic potentials in neurons of cat primary visual cortex. J Neurosci 6: 1284-1301, 1986.

Ferster D. Spatially opponent excitation and inhibition in simple cells of the cat visual cortex. J Neurosci 8: 1172-1180, 1988.

Ferster D, Chung S, and Wheat H. Orientation selectivity of thalamic input to simple cells of cat visual cortex. Nature 380: 249-252, 1996.

Ferster D and Miller KD. Neural mechanisms of orientation selectivity in the visual cortex. Аппи Rev Neurosci 23: 441-471, 2000.

Gabbott PL and Somogyi P. Quantitative distribution of GABA-immunoreactive neurons in the visual cortex (area 17) of the cat. Exp Brain Res 61: 323-331, 1986.

Gielen CC, van Gisbergen JA, and Vendrik AJ. Characterization of spatial and temporal properties of monkey LGN Y-cells. Biol Cybern 40: 157-170, 1981.

Gillespie DC, Lampl I, Anderson JS, and Ferster D. Dynamics of the orientation-tuned membrane potential response in cat primary visual cortex. Nat Neurosci 4: 1014-1019, 2001.

Hirsch JA, Alonso JM, Reid RC, and Martinez LM. Synaptic integration in striate cortical simple cells. J Neurosci 18: 9517-9528, 1998.

Hirsch JA, Martinez LM, Pillai C, Alonso JM, Wang Q, and Sommer FT. Functionally distinct inhibitory neurons at the first stage of visual cortical processing. Nat Neurosci 6: 1300-1308, 2003.

Horton JC and Sherk H. Receptive field properties in the cat's lateral geniculate nucleus in the absence of on-center retinal input. J Neurosci 4: 374-380, 1984.

Hubel DH and Wiesel TN. Receptive fields, binocular interaction and functional architecture in the cat's visual cortex. J Physiol 160: 106-154, 1962.

Hubel DH and Wiesel TN. Receptive fields and functional architecture of monkey striate cortex. J Physiol 195: 215-243, 1968.

Jones JP and Palmer LA. The two-dimensional spatial structure of simple receptive fields in cat striate cortex. J Neurophysiol 58: 1187-1211, 1987.

Kayser A, Priebe NJ, and Miller KD. Contrast-dependent nonlinearities arise locally in a model of contrast-invariant orientation tuning. $J$ Neurophysiol 85: 2130-2149, 2001.

Krukowski AE and Miller KD. Thalamocortical NMDA conductances and intracortical inhibition can explain cortical temporal tuning. Nat Neurosci 4: 424-430, 2001.

Lauritzen TZ and Miller KD. Different roles for simple-cell and complexcell inhibition in V1. J Neurosci 23: 10201-10213, 2003.

Martinez LM, Alonso JM, Reid RC, and Hirsch JA. Laminar processing of stimulus orientation in cat visual cortex. J Physiol 540: 321-333, 2002.

Mazer JA, Vinje WE, McDermott J, Schiller PH, and Gallant JL. Spatial frequency and orientation tuning dynamics in area V1. Proc Natl Acad Sci USA 99: 1645-1650, 2002.

McLaughlin D, Shapley R, Shelley M, and Wielaard DJ. A neuronal network model of macaque primary visual cortex (V1): orientation selectivity and dynamics in the input layer 4Calpha. Proc Natl Acad Sci USA 97: 8087-8092, 2000.

Mechler F and Ringach DL. On the classification of simple and complex cells. Vision Res 42: 1017-1033, 2002.

Monier C, Chavane F, Baudot P, Graham LJ, and Fregnac Y. Orientation and direction selectivity of synaptic inputs in visual cortical neurons: a diversity of combinations produces spike tuning. Neuron 37: 663-680, 2003.

Movshon JA, Thompson ID, and Tolhurst DJ. Receptive field organization of complex cells in the cat's striate cortex. J Physiol 283: 79-99, 1978a.

Movshon JA, Thompson ID, and Tolhurst DJ. Spatial and temporal contrast sensitivity of neurones in areas 17 and 18 of the cat's visual cortex. J Physiol 283: 101-120, 1978b.

Movshon JA, Thompson ID, and Tolhurst DJ. Spatial summation in the receptive fields of simple cells in the cat's striate cortex. J Physiol 283: 53-77, 1978c.

Pei X, Vidyasagar TR, Volgushev M, and Creutzfeldt OD. Receptive field analysis and orientation selectivity of postsynaptic potentials of simple cells in cat visual cortex. J Neurosci 14: 7130-7140, 1994.

Peters A and Payne BR. Numerical relationships between geniculocortical afferents and pyramidal cell modules in cat primary visual cortex. Cereb Cortex 3: 69-78, 1993.

Reid RC and Alonso JM. Specificity of monosynaptic connections from thalamus to visual cortex. Nature 378: 281-284, 1995.

Ringach DL, Hawken MJ, and Shapley R. Dynamics of orientation tuning in macaque primary visual cortex. Nature 387: 281-284, 1997. 
Ringach DL, Hawken MJ, and Shapley R. Dynamics of orientation tuning in macaque V1: the role of global and tuned suppression. J Neurophysiol 90: 342-352, 2003.

Ringach DL, Shapley RM, and Hawken MJ. Orientation selectivity in macaque V1: diversity and laminar dependence. J Neurosci 22: 5639-5651, 2002.

Saul AB and Humphrey AL. Spatial and temporal response properties of lagged and nonlagged cells in cat lateral geniculate nucleus. J Neurophysiol 64: 206-224, 1990

Schiller PH. Central connections of the retinal ON and OFF pathways. Nature 297: 580-583, 1982

Schoups A, Vogels R, Qian N, and Orban G. Practising orientation identification improves orientation coding in V1 neurons. Nature 412: 549-553, 2001.

Sclar G and Freeman RD. Orientation selectivity in the cat's striate cortex is invariant with stimulus contrast. Exp Brain Res 46: 457-461, 1982.

Series P, Latham PE, and Pouget $\mathbf{A}$. Tuning curve sharpening for orientation selectivity: coding efficiency and the impact of correlations. Nat Neurosci 7: 1129-1135, 2004.

Shapley R, Hawken M, and Ringach D. Dynamics of orientation selectivity in the primary visual cortex and the importance of cortical inhibition. Neuron 38: 689-699, 2003.

Sharon D and Grinvald A. Dynamics and constancy in cortical spatiotemporal patterns of orientation processing. Science 295: 512-515, 2002.

Sherk H and Horton JC. Receptive field properties in the cat's area 17 in the absence of on-center geniculate input. J Neurosci 4: 381-393, 1984.

Shevelev IA, Eysel UT, Lazareva NA, and Sharaev GA. The contribution of intracortical inhibition to dynamics of orientation tuning in cat striate cortex neurons. Neuroscience 84: 11-23, 1998

Shevelev IA, Sharaev GA, Lazareva NA, Novikova RV, and Tikhomirov AS. Dynamics of orientation tuning in the cat striate cortex neurons. Neuroscience 56: 865-876, 1993.

Sillito AM. The contribution of inhibitory mechanisms to the receptive field properties of neurones in the striate cortex of the cat. J Physiol 250: 305-329, 1975.

Sillito AM, Kemp JA, Milson JA, and Berardi N. A re-evaluation of the mechanisms underlying simple cell orientation selectivity. Brain Res 194: 517-520, 1980.

Skottun BC, Bradley A, Sclar G, Ohzawa I, and Freeman RD. The effects of contrast on visual orientation and spatial frequency discrimination: a comparison of single cells and behavior. J Neurophysiol 57: 773-786, 1987.
Skottun BC, De Valois RL, Grosof DH, Movshon JA, Albrecht DG, and Bonds AB. Classifying simple and complex cells on the basis of response modulation. Vision Res 31: 1079-1086, 1991.

Somers DC, Nelson SB, and Sur M. An emergent model of orientation selectivity in cat visual cortical simple cells. J Neurosci 15: 5448-5465, 1995.

Sompolinsky $\mathbf{H}$ and Shapley R. New perspectives on the mechanisms for orientation selectivity. Curr Opin Neurobiol 7: 514-522, 1997.

Stratford KJ, Tarczy-Hornoch K, Martin KA, Bannister NJ, and Jack JJ. Excitatory synaptic inputs to spiny stellate cells in cat visual cortex. Nature 382: 258-261, 1996.

Tanaka K. Cross-correlation analysis of geniculostriate neuronal relationships in cats. J Neurophysiol 49: 1303-1318, 1983.

Tao L, Shelley M, McLaughlin D, and Shapley R. An egalitarian network model for the emergence of simple and complex cells in visual cortex. Proc Natl Acad Sci USA 101: 366-371, 2004.

Teich AF and Qian N. Learning and adaptation in a recurrent model of V1 orientation selectivity. J Neurophysiol 89: 2086-2100, 2003.

Teich AF and Qian N. The modified feedforward model and the recurrent model are better suited for explaining the emergence of orientation tuning in simple and complex cells respectively. Program No. 370.8. 2004 Abstract Viewer/Itinerary Planner. Washington, DC: Society for Neuroscience, 2004, Online.

Treue S, Hol K, and Rauber HJ. Seeing multiple directions of motionphysiology and psychophysics. Nat Neurosci 3: 270-276, 2000.

Troyer TW, Krukowski AE, and Miller KD. LGN input to simple cells and contrast-invariant orientation tuning: an analysis. J Neurophysiol 87: 27412752, 2002.

Troyer TW, Krukowski AE, Priebe NJ, and Miller KD. Contrast-invariant orientation tuning in cat visual cortex: thalamocortical input tuning and correlation-based intracortical connectivity. J Neurosci 18: 5908-5927, 1998.

Tsumoto T, Eckart W, and Creutzfeldt OD. Modification of orientation sensitivity of cat visual cortex neurons by removal of GABA-mediated inhibition. Exp Brain Res 34: 351-363, 1979

Vidyasagar TR and Siguenza JA. Relationship between orientation tuning and spatial frequency in neurones of cat area 17. Exp Brain Res 57: $628-631,1985$.

Wielaard DJ, Shelley M, McLaughlin D, and Shapley R. How simple cells are made in a nonlinear network model of the visual cortex. J Neurosci 21: 5203-5211, 2001. 